\title{
Zur Geschichte der Gleichung des V. Grades (bis 1858).
}

Von J. Pierpont in Wien.

\section{Einleitung.}

Nach den glänzenden Erfolgen der italienischen Mathematiker des XVI. Jahrhunderts konnten die Zeitgenossen von Ferro, Tartaglia, Cardano und Ferrari wohl glauben, dass die Auflösung der allgemeinen Gleichung fünften Grades bald erfolgen würde. Es vergieng jedoch mehr als ein Jahrhundert, ehe man überhaupt eine Methode ersann, die auf diese Gleichung anwendbar war. Erst im Jahre 1683 in den "Acta Eruditorum" erschien eine Abhandlung über diesen Gegenstand von dem Naturforscher und Philosophen Tschirnhaus. Diese Abhandlung blieb die einzige einschlägige im XVII. Jahrhundert, während des XVIII. aber wurde die Auflösung der allgemeinen Gleichung fünften Grades geradezu eines der berühmten mathematischen Probleme des ganzen Jahrhunderts; Euler, Bézout, Lagrange, Van. dermonde und Malfatti widmeten demselben eingehende Untersuchungen. Ihre Arbeiten, obschon reich an wertvollen Resultaten, waren naturgemäl in der Hauptsache ohne Erfolg. Die Hoffnung eines Leibnitz und Ts chirn ha us machte einem ernsten Zweifel Platz. Die Resolventen vierundzwanzigsten Grades, deren Auflösung die Methoden von Tschirnhaus, Euler und Bézout verlangten, wurden allerdings durch die Untersuchungen von Lagrange, Vandermonde und Malfatti durch eine vom sechsten Grade ersetzt. Eine weitere Reduction schien aber aussichtslos, und die Auflösung der allgemeinen Gleichung fünften Grades somit ein Ding der Unmöglichkeit.

Es war ein Italiener, namens $R$ uffini, Arzt und Mathematiker, der am Schluss des Jahrhunderts die kühne Idee fasste, dies zu beweisen. Sein Werk, das 1799 zu Bologna unter dem Titel "Teoria generale delle Equazioni" erschien, wird für immer einen Markstein in der Geschichte der algebraischen Gleichungen bilden. Dass ein so dornenvolles Unternehmen im ersten Verlaufe völlig gelingen würde, war kaum zu erwarten und erst dem jugend- 
lichen Abel war es vorbehalten, einen vollkommen strengen Beweis zu liefern, und so für das Problem der Auflösung dieser Gleichung eine richtige Fragestellung vorzubereiten.

Zwei neue Zweige der Mathematik sollten nunmehr in dieser Frage die Hauptrolle spielen; einerseits die durch Abel und Jacobi ausgebildete Theorie der elliptischen Functionen, anderseits die von Galois angebahnte Gruppentheorie. Die ersteren gelangten zu gewissen algebraischen Gleichungen vom sechsten Grade, deren Wurzeln bekannte elliptische Functionen waren; der letztere zeigte, dass die Gruppe dieser Gleichungen und die Gruppe der Resolventen sechsten Grades der allgemeinen Gleichung fünften Grades holoedrisch isomorph waren. Diese Resultate wurden sodann auf verschiedene Weise von Hermite und Kronecker verwendet. Hermite zeigte, wie die Resolvente fünften Grades der Modulargleichung sechsten Grades mit der allgemeinen Gleichung fünften Grades verbunden werden könne; Kronecker, der im Gegensatze zu Hermite sich fast ganz auf dem Gebiete der Algebra bewegte, zeigte, wie man eine rationale Resolvente vom sechsten Grade der allgemeinen Gleichung fünften Grades finden könne, deren Coefficienten von nur zwei Parametern abhiengen, und die wegen ihrer merkwürdigen Eigenschaften geeignet war, als Normalresolvente zu gelten.

Eine zusammenhängende Darstellung der auf die Gleichung V. Grades bezüglichen Untersuchungen zu liefern, welche bis zum Einde des Jahres 1858 erschienen, ist der Zweck der folgenden Zeilen.

\section{Tschirnhaus.}

Der erste Versuch einer algebraischen Auflösung der allgemeinen Gleichung fünften Grades wurde, wie wir schon bemerkt haben, von Tschirnhaus gemacht, der im Jahre 1683 in den "Acta Eruditorum" eine Abhandlung mit dem Titel "Methodus Auferendi Omnes Terminos Intermedios ex Data Aequatione" veröffentlichte. Es beginnt mit folgenden Worten:

nEx Geometria Dn. Des Cartes notum est, qua ratione semper secundus terminus ex data aequatione possit auferri; quod plures terminos intermedios auferendos hactenus nihil inventum vidi in Arte Analytica, imo non paucos offendi, qui crediderunt, id nulla arte perfici posse. Qua propter hic quaedam circa hoc negotium aperire constitui, verum saltem pro is qui Artis Analyticae apprime gnari cum aliis tam brevi explicatione vix satisfieri possit reliqua, quae hie desiderari possent alli tempori reservans".

Tschirnhaus' Entdeckung besteht nun in Folgendem:

Er bemerkte, dass die Hilfsgleichung

$$
x=y+a
$$


welche Descartes benützt, um $x$ aus der gegebenen Gleichung zu eliminieren, eine einzige Constante $a$ enthält, und wurde dadurch auf die Idee geführt, eine neue Hilfsgleichung

$$
x^{2}=b x+y+a
$$

zu nehmen. Da diese zwei willkürliche Constanten enthält, so wird man imstande sein, zwei Glieder der gegebenen Gleichung zu entfernen. Dass dies wirklich so sei, zeigte er durch Anwendung auf Gleichungen bis zum sechsten Grade. Gleichung

Nunmehr ist es leicht zu bemerken, dass man vermittelst der

$$
x^{3}=c x^{2}+b x+y+a
$$

3 Glieder einer Gleichung entfernen könne u. s. f.

Darauf bemerkt Tsehirnhaus:

\#... qualis autem promotio, Analyseos haec fit et quanti usus periti Analystae facile judicabunt: Ego unicum saltem hic corollarium deducam, quod numerum huic Methodus exhibeatur omnium aequationum cujuscumque gradus Radices analytice determinandi, idque exemplo ostendam".

Wie sehr Tschirnhaus seine Methode überschätzt, wird durch folgende Stelle klar:

"Notandum vero ultimo, quod non solum ope aequationum exhibitarum duo tres quatuor etc. termini ex data aequatione auferri possint, sed quod variis aliis aequationibus, diversis ab his, idem quoque facere possim; imo quod omnes possibiles aequationes ope quarum hoc negotium peragi possit, facile enumerare veleam; ac quod proinde omnium aequationum omnes possibiles expressiones Analyticas Radicum, hac Methodo exhibere possim prout suo loco diffusius explicabo".

Als Beispiel dieser neuen Gleichungen führt er an:

$$
y z=z^{2}+a
$$

die er dann anwendet, um die cubische Gleichung aufzulösen.

Tschirnhaus' Methode führt leicht und direct zu der Auflösung der quadratischen und cubischen Gleichungen und ist in dieser Beziehung ein Fortschritt. Jedoch schon für die biquadratische Gleichung wird die Arbeit betriichtlich, während sie im Falle der. Gleichung fünften Grades beinahe nicht zu bewältigen ist. Dies ist wohl auch der Grund, weshalb Tschirnhaus keinen Versuch 
machte, seine Methode auf dieselbe anzuwenden, sondern sich von deren Erfolg durch Induction überzeugte, eine Sitte, die zu seiner Zeit unter den besten Mathematikern nur zu sehr verbreitet war.

Wie wir sehen werden, zeigte Lagrange, dass diese Methode stets auf Gleichungen von höherem Grade als dem der gegebenen Gleichung führt, sobald der Grad der letzteren größer als 4 ist.

Es dürfte hier der geeignete Platz sein, die weitere Geschichte der Tschirnhaus'schen Transformation zu geben, insofern sie die Gleichung fünften Grades berührt. Ein schwedischer Mathematiker, Bring, zeigte in einer zu Lund 1786 veröffentlichten Schrift ${ }^{1}$ ), wie man vermittelst einer solchen Transformation, die allgemeine Gleichung fünften Grades mit Hilfe einer cubischen Gleichung auf die Form

$$
x^{5}+a x+b=0
$$

zuzückführen könne, welche nur einen Parameter enthält. Dieses Resultat, welches längst vergessen war, wurde von Jerrard neu entdeckt und in seinen "Mathematical Researches" 1834 veröffentlicht. Man nannte deswegen lange Zeit die obige Form die Jerrard'sche, bis Hill 1861 in den Abhandlungen der schwedischen Akademie die Rechte seines Landsmannes zur Geltung brachte. Hermite's Auflösung der allgemeinen Gleichung fünften Grades durch elliptische Functionen hängt von der Möglichkeit einer solchen Transformation ab. Er nennt sie "le pas le plus important qui ait été fait dans la théorie algébrique des équations du cinquième degré depuis qu'Abel a démontré qu'il était impossible de les resoudre par radicaux. ${ }^{4}$

Ich will nun eine kurze Skizze von Bring's Methode geben. Durch die Auflösung einer quadratischen Gleichung können wir die allgemeine Gleichung fünften Grades, wie Tschirnhaus gezeigt hat, auf die Form

$$
z^{5}+p z^{2}+q z+r=0
$$

bringen.

Wir setzen nun

$$
z^{4}+d z^{3}+c z^{2}+b z+a+y=0
$$

und eliminieren $\approx$; dadurch erhalten wir

1) Meletemata quaedam mathematica circa transformationem aequationum algebraicarum.

Harley: Quarterly Journal of Mathematics. Bd. 6. (1863).

Grunerts Archiv der Mathematik und Physik. Bd. 41, S. 105. 


$$
y^{5}+(5 a-4 q-3 p d) y^{4}+
$$

$+\left(3 p b c+4 q b d+5 r b+2 q c^{2}+5 r c d-3 p^{2} c+6 q^{2}-4 p r+\right.$ $\left.+5 q p d+3 p^{2} d^{2}-12 p d a-16 q a+10 a^{2}\right) y^{2}+$

$+1-p b^{3}-4 q b^{2} c-5 r b^{2} d+3 p^{2} b^{2}+9 p b c a+12 q b d a-$ $-5 r b c^{2}-3 p^{2} b c d+2 p q b c-5 p q b d^{2}+15 r b a+$ $+\left(p r-8 q^{2}\right) b d-\left(11 r q-3 p^{3}\right) b+6 q c^{2} a+15 r c d a+$

(3)

$$
\begin{aligned}
& +p^{2} c^{3}+p q c^{2} d+8\left(r p-4 q^{2}\right) c^{2}-9 p^{2} c a+18 q^{2} a+ \\
& +\left(4 q^{2}-7 p r\right) c d^{2}-\left(2 q r+3 p^{3}\right) c d-12 p r a+15 p q d a+ \\
& +\left(2 p^{2} q+5 r^{2}\right) c-\left(p^{3}+3 r q\right) d^{3}+9 p^{2} d^{2} a-\left(q p^{2}+5 r^{2}\right) d^{2}- \\
& -\left(p q^{2}+r p^{2}\right) d-18 p d a^{2}+p^{4}-4 q^{3}+8 r p q+10 a^{3}- \\
& +\ldots=0 . \\
& +24 q a^{2}
\end{aligned}
$$

Bezeichnen wir die Coefficienten des zweiten, dritten und vierten Gliedes von (3) resp. mit $A, B, C$, so gibt die Gleichung $A=0$

$$
a=\frac{3 p d+4 q}{5}
$$

und wenn wir

$$
b=\alpha d+\zeta \quad c=d+\gamma
$$

sêtzen und diese Werte in $B=0$ einsetzen, erhalten wir

$$
\begin{gathered}
\left\{(15 p+20 q) \alpha-3 p^{2}+10 q+25 r\right\} d^{2} \\
+\left\{(15 p \alpha+20 q+25 r) \gamma+(15 p+20 q) \zeta+25 r \alpha-15 p^{2}-23 p q\right\} d \\
+10 q \gamma^{2}+\left(15 p \zeta-15 p^{2}\right) \gamma+25 r \zeta-2 q^{2}-2 r p=0
\end{gathered}
$$

Bestimmen wir nun $\alpha$ and $\zeta$ derart, dass die Coefficienten der ersten 2 Glieder dieser Gleichung 0 sind, so erhalten wir:

$$
\begin{gathered}
\alpha=\frac{3 p^{2}-10 q-25 r}{15 p+20 q} \\
\zeta=\frac{15 p \alpha \gamma-25 r \alpha-20 q \gamma-25 r \gamma+15 p^{2}+23 p q}{15 p+20 q} \\
10 q \gamma^{2}-\left(15 p^{2}-15 p \zeta\right) \gamma+25 r \zeta-2 q^{2}-2 r p=0 .
\end{gathered}
$$

Setzen wir die durch die Gleichungen (4), (5), (6) bestimmten Werte von $a, b, c$ in $C=0$ ein, so entsteht eine cubische Gleichung in $d$. Somit ist die Transformation (2) bestimmt und (1) nimmt die Form an:

$$
y^{5}+a y+b=0
$$




\section{Euler.}

Wir wollen nun zwei hierher gehörige Abhandlungen von Euler betrachten.

In der ersten, ${ }^{1}$ ) welche 1732 veröffentlicht wurde, drückt Euler gleich am Anfange sein Erstaunen über den geringen Erfolg der Mathematiker betreffs der Auflösung der höheren Gleichungen aus. Er schreibt: „Summopere admirandum videtur, quod cum ipsis rei analyticae initiis radices aequationum cubicarum et biquadricarum essent inventae, his tamen temporibus, quibus analysis maxima augmenta accepit, modus adhuc lateat altiorum aequationum radices eruendi praesertim cum haec res continuo a praestantissimis ingeniis maximi studio sit investigata."

Fr schlägt nun folgende Gestalt der Wurzel vor

$$
x=\sqrt[n]{\bar{A}}+\sqrt[n]{\bar{B}}+\bar{v}^{n}+\ldots
$$

denn ist das zweite Glied einer Gleichung durch Descartes' Transformation entfernt, so hat die Wurzel der Gleichungen. zweiten, dritten, vierten Grades resp. folgende Form :

$$
\begin{aligned}
& x=\sqrt[V]{ } \\
& x=\sqrt[3]{A}+\sqrt[3]{B} \\
& x=\sqrt[4]{A}+\sqrt[4]{B}+\sqrt{C}
\end{aligned}
$$

wie er thatsächlich zeigt. Die Größen $A, B, C$ sind Wurzeln einer Gleichung, deren Grad um 1 kleiner ist, als der Grad der zugehörigen gegebenen Gleichung. Diese Gleichung, von deren Auflösung die der gegebenen Gleichung abhängt, nennt er die resolvierende Gleichung (aequatio resolvens), so dass Euler, wie es scheint, der Erfinder dieses Ausdruckes ist. Sobald nun der Grad $n$ der gegebenen Gleichung 4 übersteigt, ist Euler nicht imstande, die Resolvente vom $n-1^{\text {ten }}$ Grade zu finden.

"Tamen", so bemerkt er, ,praesto sunt non nullius momenti argumenta, quibus ista mea conjectura confirmatur. Si enim. aequatio proposita ita est comparata ut in aequatione resolvente omnes termini praeter tres primos evanescant; tum semper aequatio resolvens poterit exhiberi atque ideo aequationis propositae radices assignari. Aequationes autem, quae hoc modo resolutionem admittunt, sunt eae ipsae, quos Cl. Abr. de Moivre in Transact. No. 309 pertractavit."

1) Conjectio de formis radicum aequationtum cujusvis ordinis Com. Petrop. Bd. 6. $1732-3$. 
Dreibig Jahre später, im Jahre 1762, nimmt Euler das Problem nochmals in Angriff ${ }^{1}$ ) und schlägt eine zweite Form für die Wurzeln vor. Über die ältere Form bemerkt er: "Quam conjecturam confirmavi, ostendens resolutionem aequationem inferiorum revera ex hoc forma generali deduci, neque etiam nune dubito quin haec conjectura veritati sit consentanea."

Es ist natürlich äußerst wichtig, die Wurzelform möglichst genau zu präcisieren; gewisse Schwierigkelten, woran die ältere Methode leidet, werden wohl deshalb entstehen, weil in dem Ausdruck

$$
x=\bar{v} \bar{A}+\ddot{\bar{v}} \bar{B}+\ldots
$$

der Wert der Wurzel nicht bestimmt ist. Er schlägt daher die folgende neue Form vor:

$$
x=a_{0}+a_{1} \sqrt[n]{v}+a_{2} \sqrt{v}^{2}+\ldots+a_{n-1} \sqrt{v}^{n} v^{n-1},
$$

wo $v, a_{1}, a_{2} \ldots$, entweder rationale Functionen der Coefficienten sind oder mindestens keine $x^{\text {te }}$ Wurzel enthalten. Die übrigen Wurzeln der Gleichung sind nunmehr vollkommen bestimmt; wir erhalten sie, indem wir der Größue $\bar{v} \bar{v}$ die Werte

$$
\sqrt[n]{v}, \quad \alpha \stackrel{n}{v}, \quad \alpha^{2} \ddot{v}_{v} \ldots \alpha^{n-1} \dot{v}^{n}
$$

beilegen, wo $\alpha$ eine primitive $n^{\text {te }}$ Einheitswurzel ist.

Euler zeigt, dass die Wurzeln der Gleichung zweiten, dritten und vierten Grades unter diese Form gebracht werden können.

Wir wollen seine Methode durch ein Beispiel erläutern:

Ist

$$
x^{3}=A x+B
$$

die vorgelegte Gleichung, so haben nach Euler deren Wurzeln die Form:

$$
\begin{aligned}
& x_{1}=a_{1} \sqrt[3]{v}+a_{2} \sqrt[3]{v^{2}} \\
& x_{2}=a_{1} \alpha \sqrt[3]{v}+a_{2} \alpha^{2} \sqrt[3]{v^{2}} \quad\left(a^{3}-1=0\right) \\
& x_{3}=a_{1} \alpha^{2} \sqrt{v}+a_{2} \alpha \sqrt[3]{v^{2}}
\end{aligned}
$$

Die erste Gleichung gibt

$$
x^{3}=a_{1}^{3} v+3 a_{1}^{2} a_{2} v \bar{V}^{3} \bar{v}+3 a_{1} a_{2}^{2} v \bar{v}^{2}+a_{2}^{3} v^{2} .
$$

1) De resolutione aequationum cujusvis gradus. Nov. Com, Petrop. Bd. 9. 
Durch Vergleich mit (1) erhalten wir

$$
a_{2}=\frac{A}{3 v}, \quad v^{2}=B v-\frac{A^{3}}{27},
$$

wenn wir, was gestattet ist, $a_{1}=1$ setzen. Somit sind $a_{1}, a_{2}, v$ bestimmt und damit auch die Wurzeln $x_{1}, x_{2}, x_{3}$.

Die Anwendung dieser Methode aut die Gleichung fünften Grades führt Euler auf eine Gleichung vom vierundzwanzigsten Grade.

In der That; es sei die vorgelegte Gleichung

$$
x^{5}+a x^{3}+b x^{2}+c x+d=0
$$

und

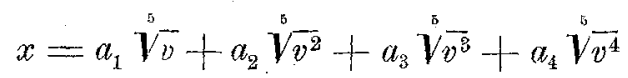

eine Wurzel derselben. Wenn wir $a, b, c, d$ durch die Größen. $s_{1}, s_{2}, \ldots$ ausdrücken, wo $s_{*}=\sum_{\lambda=1}^{\lambda=5} x_{\lambda}^{*}$ ist, so erhalten wir vier Gleichungen:

$$
a=\varphi_{1}, \quad b=\varphi_{2}, \quad c=\varphi_{3} \quad d=\varphi_{+},
$$

wo die $\varphi$ resp. vom ersten, zweiten, dritten und vierten Grade in $v$, und vom zweiten, dritten, vierten und fünften Grade in $a_{1}, a_{2}, a_{3}, a_{4}$ sind.

Euler bemerkt nun:

„Si nunc vicissim ex datis coefficientibus $a, b, c, d$ definiri possent quantitates $a_{1}, a_{2}, a_{3}, a_{4}$ cum littera $v$ haberetur resolutio generalis omnium aequationum quinti gradus. Verum in hoe ipso summa difficultas consistit, cum nulla via pateat litteras $a_{1}, a_{2}, a_{3}, a_{4}$ quarum quidem unam pro libitu assumere licet, successive ita eliminandi, ut aequatio solum incognitam $v$ cum datis $a b c d$ involvens resultet, quae quidem nullas radices superfluas complectatur. Satis tuto autem suspicari licet, si haec eliminatio rite administretur, tandem ad aequationem quarti gradus perveniri posse, qua valor ipsius $v$ definiatur. Si enim aequatio altioris gradus proderit, tum quoque valor ipsius $v$ signa radicalia ejusdem gradus implicaret, quod absurdum videtur."

Euler's Schlussweise ist hier ganz correct; der Fehler liegt nur in seiner Voraussetzung, dass die Größen $v, a_{1} a_{2} \ldots$ algebraisch sind. Lagrange, der Euler's Methode untersuchte, obschon er zu einem anderen Resultat als Euler betreffs des Grades der Resolvente kam, vermochte Euler's Irrthum nicht aufzuklären. 
Die zweite Euler'sche Form, in etwas präciserer Fassung, bildet die Grundlage von Abel's Beweis der Unmöglichkeit der algebraischen Auflösung der allgemeinen Gleichung $n^{\text {ten }}$ Grades $(n>4)$; es dürfte deswegen die Bemerkung nicht ohne Interesse sein, dass Euler auf dieselbe durch die Bemerkung geführt wurde, dass die $n^{\text {ten }}$ Einheitswurzeln durch

$$
a, a^{2}, \alpha^{3}, \ldots, \alpha^{n-1}, a^{n} \quad\left(\alpha^{n}=1\right)
$$

dargestellt werden können und dass die Fortsetzung dieser Reihe

$$
\alpha^{n+1}, \alpha^{n+2} \ldots
$$

nur dieselben Größen hervorbringt.

Er vermuthete deswegen, dass, abgesehen von Factoren, welche keine $n^{\text {te }}$ Wurzel enthalten, die einzelnen Glieder des Wurzelausdruckes, Potenzen eines einzigen unter ihnen sind,

$$
\stackrel{n}{V}^{n}, \ddot{n}^{2} v^{2} \ldots \stackrel{n}{V}^{n-1}, \ddot{V}^{n}, \ddot{V}^{n+1} v^{n+1} v^{n+2} \ldots
$$

$\mathrm{Da} \ddot{V}^{n}=v$, so enthält der Wurzelausdruck im allgemeinen ein Glied, welches von dem Wurzelzeichen frei ist; und da

$$
\grave{v}^{n+1}=v \ddot{v}^{n} v, \quad \hat{\boldsymbol{V}} v^{n+2}=v \ddot{\boldsymbol{V}}^{2}
$$

ist, so wird der Wurzelausdruck die Form haben:

$$
x=a_{0}+a_{1} \ddot{v}^{n} v+\ldots a_{n-1} \grave{\eta}^{n-1}
$$

und es sind die übrigen Wurzeln nun eindeutig bestimmt.

Angesichts der Thatsache, dass die Wurzeln aller damals bekannten auflösbaren Gleichungen die obige Form haben, gewinnt Euler die volle Überzeugung der Richtigkeit seiner Annahme. "His igitur argumentis", sagt er, "nova haec radicum forma jam ad summum probabilitatis est evecta."

\section{Bézout.}

Ungefähr gleichzeitig mit Euler gelangte Bézout ${ }^{1}$ ) zu ähnlichęn Resultaten.

Wir wollen seine Methode durch ein Beispiel erläutern:

1) Sur plusieurs classes d'équations de tous les degrés qui admettent une solution algébrique. Hist. dé l'Acad. des Sciences. Paris 1762.

Sur la resolution générale des équations de tous le degrés. $\mathbf{1 7 6 5 .}$ 
Es sei

$$
x^{3}+p x+q=0
$$

eine vorgelegte Gleichung. Wir setzen

$$
\begin{gathered}
y^{3}-1=0 \\
a y^{2}+b y+x=0
\end{gathered}
$$

und eliminieren $y$, indem wir (2) mit $y$ und $y^{2}$ multiplicieren, wodurch das System von linearen Gleichungen:

$$
\begin{aligned}
& a y^{2}+b y+x=0 \\
& b y^{2}+x y+a=0 \\
& x y^{2}+a y+b=0
\end{aligned}
$$

entsteht, aus welchem sich die Gleichung:

$$
\left|\begin{array}{lll}
a & b & x \\
b & x & a \\
x & a & b
\end{array}\right|=0=x^{3}-3 a b x+a^{3}+b^{3}
$$

ergibt.

Durch Vergleichung mit (1) erhalten wir:

$$
3 a b=-p \quad a^{3}+b^{3}=q
$$

also

$$
a^{6}-q a^{3}-\frac{p^{3}}{27}=0 .
$$

Ist nun $\alpha$ eine primitive dritte Einheitswurzel, so sind die 3 Wurzeln von (1):

$$
\begin{aligned}
& -x_{0}=a b+a^{2} a \\
& -x_{1}=a^{2} b+\alpha a \\
& -x_{2}=b+a .
\end{aligned}
$$

Diese Methode, auf die Gleichung fünften Grades angewendet, führt wie bei Euler auf eine Resolvente vom vierundzwanzigsten Grade. Die Methoden von Euler und Bézout sind in der That in gewissem Sinne sehr ähnlich. Beide nehmen an:

$$
x=a_{0}+a_{1} \bar{v}_{v}+a_{2} \bar{n}_{v^{2}}+\ldots
$$

jedoch setzt Euler $a_{1}=1$, während Bézout $v=1$ nimmt. Bézout bemerkt nun: "On verra par la comparaison du Mémoire de M. Euler avec le mien, que quoique nos deux méthodes puissent 
conduire aux même résultats, nous différont eependant beaucoup sur l'estimation du degré de l'équation dont doit dependre la résolution de l'équation proposée. Ce savant Analyst pense qu'il sera toujour's moindre que celui de la proposée, je pense au contraire qu'il sera toujours beaucoup plus élevé, mais que l'equation ne renfermera que les difficultés de tous les degrés inférieurs à la proposée".

Den wahren Unterschied zwischen seiner und Euler's Methode. hat Bézout, wie wir sehen, also nicht bemerkt. Nach Bézout's Voraussetzung haben die Wurzeln die Form

$$
\begin{aligned}
& x=a+b+c+\ldots . \\
& x_{1}=a+\alpha b+a^{2} c+\ldots . \quad\left(\alpha^{n}=1\right)
\end{aligned}
$$

wo $a, b, c .$. . . unbestimmte Größuen sind; nach Euler's Annahme dagegen sind sie algebraisch.

\section{Lagrange.}

Lagrange's Abhandlung ,Sur la résolution algébrique des Equations"1) ist dadurch merkwürdig, dass in derselben zum erstenmal rationale Functionen der Wurzeln eingeführt, die Werte, die solche Functionen durch Vertauschung der Wurzeln annehmen, untersucht und die Resultate systematisch verwertet werden, um die algebraische Auflösung der Gleichungen zu bewerkstelligen. Wir können den Wert dieser Abhandlung kaum überschätzen, denn in ihr fanden Ruffini, Abel und Galois die Keime ihrer großen Methoden vor. Nachdem Euler eine allgemeine Methode der Auflösung der Gleichungen von den ersten vier Graden entdeckt hatte, schloss er auf dem Wege der Induction, wie wir gesehen haben, dass dieselbe zur Aufösung der Gleichungen aller Grade benützt werden könnte. Lagrange beabsichtigte nun eine noch weitere Induction zu machen. Er analysierte alle damals bekannten Methoden der Auflösung, entdeckte ihr gemeinsames Princip und suchte dasselbe für die Auflösung der allgemeinen Gleichung zu verwerten. Da seine Methoden ohne den gew ünschten Erfolg blieben, gab er die Gründe für dieses Fehlschlagen an.

Als Beispiel soll seine Analyse der Methoden von Tse hirnhaus, Euler und Bézout auseinandergesetzt werden.

Um die Gleichung

$$
x^{n}+a_{1} x^{n-1}+\ldots+a_{n}=0
$$

aufzulösen, setzt man

$$
y=p_{0}+p_{1} x+\cdots \cdot+p_{n-1} x^{n-1}
$$

1) Mem. d. Akad. d. Wiss. zu Berlin 1770-71. 
und es ergibt sich

und

$$
y^{n}+b_{1} y^{n-1}+\ldots+b_{n}=0
$$

$$
x=q_{0}+q_{1} y+\cdots+q_{n-1} y^{n-1} .
$$

Wir setzen nun

$$
b_{1}=b_{2}=\ldots \cdot=b_{n-1}=0
$$

so dass nunmehr

wird.

$$
y^{n}+b_{n}=0
$$

Nehmen wir jetzt $b_{n}=-1$, so haben wir

$$
\begin{aligned}
& y^{n}-1=0 \\
& x=q_{0}+q_{1} y+\ldots .+q_{n-1} y^{n-1},
\end{aligned}
$$

was Bézout's Annahme ist.

Hätten wir $q_{1}=1$ gesetzt, so hätten wir erhalten

$$
x=q_{0}+\sqrt[n]{v}+q_{2} \sqrt[n]{v^{2}}+\ldots .+q_{n-1} \sqrt[n]{v^{n-1}},
$$

wo $v=-b_{n}$ ist, was Euler's Annahme ist.

Diese Betrachtungen zeigen das gegenseitige Verhältnis dieser Aufösungsmethoden vollkommen deutlich. Wir verfolgen nun eine dieser Methoden weiter; da Bézout's Form etwas einfacher ist, wollen wir diese wählen. Es ist

$$
\begin{aligned}
& x_{0}=q_{0}+q_{1}+\ldots+q_{n-1} \\
& x_{1}=q_{0}+a q_{1}+\ldots .+a^{n-1} q_{n-1} \quad\left(a^{n}-1=0\right)
\end{aligned}
$$

somit ergeben sich die Gleichungen:

$$
\begin{aligned}
& n q_{0}=x_{0}+x_{1}+\ldots+x_{n-1} \\
& n q_{1}=x_{0}+\alpha^{-1} x_{1}+\ldots+\alpha^{-(n-1)} x_{n-1}
\end{aligned}
$$

Die Bestimmung der $q$ hängt also von der Function:

$$
t=x_{0}+\alpha x_{1}-+\alpha^{2} x_{2}+\ldots .+\alpha^{n-1} x_{n-1}
$$

ab. 
Durch eine ähnliche Analyse zeigte Lagrange, dass alle damals bekannten Aufösungsmethoden von einer solchen Function abhängen.

Diese Function $t$ ist Wurzel einer irreductiblen Gleichung: vom Grade $n$ !

$$
\theta(t)=\left(t-t_{0}\right)\left(t-t_{1}\right)\left(t-t_{2}\right) \ldots .=0
$$

Durch das Studium der Auflösung dieser Gleichung für $n<5$ zeigte Lagrange, wie dieselbe für den allgemeinen Fall behandelt werden könne.

Zuerst bemerkte Lagrange, dass die Gröbe $t$ in den $\alpha^{0} x_{0}$, $\alpha x_{1}, \alpha^{2} x_{2}$. . symmetrisch ist, so dass also eine Vertauschung von $x_{0}, x_{1}, \ldots$. dieselbe Wirkung hat, wie eine Vertauschung: von $\alpha^{0}, \alpha, \alpha^{2}, \ldots$.

Wendet man nun die cyklische Substitution

$$
s=\left(x_{0}, x_{1} \cdot \ldots \cdot x_{n-1}\right)
$$

auf $t$ an, so geht es in $\alpha^{-1} t$ über, bei $s^{2}$ in $a^{-2} t$, bei $s^{3}$ in $a^{-3} t$ u. s. f.

Somit sind

$$
t, \alpha t, \alpha^{2} t . . \cdot \alpha^{n-1} t
$$

Wurzeln der Gleichung $\theta(t)=0$, und dieselbe enthält nur Glieder, deren Exponenten ein Vielfaches von $n$ sind.

Wir setzen daher $t^{n}=0$ und bezeichnen mit

$$
\theta_{1}, \theta_{2} \cdots \theta_{n-1}
$$

die Werte von $\theta$, nachdern $\alpha$ bez. durch $\alpha, \alpha^{2}, \alpha^{3} \cdots \alpha^{n-1}$ ersetzt wurde.

Es sei nun

$$
\begin{gathered}
\theta^{n-1}+\mathrm{T} \theta^{n-2}+\mathrm{U} \theta^{n-3}+\ldots+\mathrm{X} \theta+\mathrm{Y}=\left(\theta-\theta_{1}\right)\left(\theta-\theta_{2}\right) \\
\cdots\left(\theta-\theta_{n-1}\right)=0 .
\end{gathered}
$$

Die Auflösung der vorgelegten Gleichung (1) hängt von der Auflösung von (2) ab; denn wir haben

$$
\begin{array}{rrl}
x_{0}+x_{1}+\ldots+x_{n-1} & =-a_{1}=\sqrt[n]{\theta_{0}} \\
x_{0}+a x_{1}+\ldots+\alpha^{n-1} x_{n-1} & =\sqrt[n]{\theta_{1}} \\
\cdot & \cdots & \cdots \\
\cdot & \cdots & \cdots \\
\cdot & \cdots & \cdots \\
x_{0}+\alpha^{n-1} x_{1}+\ldots & & =\sqrt[n]{\theta_{n-1}}
\end{array}
$$


und somit z. B.

$$
x_{0}=\frac{\sqrt[n]{\theta_{0}}+\sqrt[n]{\theta_{1}}+\ldots+\sqrt[n]{\theta_{n-1}}}{n}
$$

Wir bemerken in Vorübergehen, dass dies Euler's erste Form ist.

Da die Gleichung (2) nur vom $n-1^{\text {ten }}$ Grade ist, so ist dieselbe immer auflösbar, wenn nur die Coefficienten $T, U, \ldots X, \mathrm{I}^{\circ}$ bestimmt werder können.

Es bieten sich nun zwei Fälle dar, jenachdem $n$ eine Prim-, oder zusammengesetzte Zahl ist.

Es sei zuerst $n$ eine Primzahl. Lagrange bemerkt, dass man alle $(n-1)$ ! Werte von $\theta$ erhält, wenn man die $n-2$ Wurzeln $x_{2} x_{3} \ldots x_{n-1}$ auf alle möglichen Weisen vertauscht und in jedem davon $\alpha$ dureh $\alpha^{2} \alpha^{3} \ldots \alpha^{n-1}$ ersetzt.

Eine rationale symmetrische Function $\rho, \operatorname{der} \theta_{1}, \theta_{2}, \ldots, \theta_{n-1}$ ist somit Wurzel einer Gleichung vom Grade $p=(n-2)$ ! Die Coefficienten $\mathrm{T}, \mathrm{U}, \ldots$ sind rational in $\varphi$. Wie Lagrange bemerkt, zerlegt sich $\theta=0$ in $(n-2)$ ! Factoren vom Grade $n-1$, sobald $\operatorname{man} \varphi$ als bekannt ansieht.

Für den Fall, dass $n$ eine zusammengesetzte Zahl ist, haben die vorhergehenden Betrachtungen keine Anwendung. In diesem Falle zeigt Lagrange, wie man die gegebene Gleichung (1) in $p$ Gleichungen vom $m^{\text {ten }}$ Grade zerlegen kann, wo $p$ ein Primfactor von $n$ ist.

"Wir bilden die folgende Tabelle:

$$
\begin{aligned}
& x_{0} \quad x_{p} \quad \ldots x_{(m-1) p} \\
& x_{1} \quad x_{p+1} \ldots x_{(m-1) p+1} \quad(n=m p) \\
& \ldots \ldots \ldots \ldots \cdots \cdots \cdots \cdots \\
& x_{p-1} x_{2 p-1} \ldots x_{m p-1}
\end{aligned}
$$

Es mögen die Summen der Elemente einer Reihe resp. mit

$$
X_{1}, X_{2}, \ldots, X_{p}
$$

-bezeichnet werden. Wir betrachten eine Gleichung, deren Wurzeln die Elemente einer Reihe sind, etwa die der ersten:

$$
F_{1}=\left(x-x_{0}\right)\left(x-x_{p}\right) \ldots\left(x-x_{(m-1) p}\right)=0 .
$$

Die Coefficienten dieser Gleichung sind rationale Functionen der Größe $X_{1}$. Das entsprechende gilt für die Gleichungen

$$
\mathrm{F}_{2}=0, \mathrm{~F}_{3}=0, \ldots, \mathrm{F}_{p} \stackrel{\rho}{=}
$$


deren Coefficienten in

rational sind.

$$
X_{2} \text { bez. } X_{3} \ldots \text { bez. } X_{p}
$$

Sind diese Größen nun bekannt, so haben wir $p$ Gleichungen: vom $m^{\text {ten }}$ Grade mit rationalen Coefficienten.

Wir bilden also die Gleichung:

$$
X=\left(X-X_{1}\right) \ldots\left(X-X_{p}\right)=0 \text {. }
$$

Die Coefficienten dieser Gleichung hängen von einer Größe ९. $a b$, die einer Gleichung vom Grade $\rho=\frac{n !}{p !(m !)^{p}}$ genügt. In der That, sei $\varphi$ eine rationale symmetrische Function der Größen $X_{1}, X_{2}, \ldots X_{p}$. Eine Vertauschung der Elemente einer Reihe der Tabelle $(a)$ ändert den Wert von $\varphi$ nicht; ferner, ändert eine Vertauschung der $x$, welche blol eine Permutation der $X$ hervorbringt, ebenfalls den Wert von $\varphi$ nicht. Es sind also $p !(m !)^{p}$ Substitutionen, welche $\varphi$ unverändert lassen; somit ist die Anzahl $\rho$ der conjugierten $\varphi=\frac{n !}{p !(m !)^{p^{*}}}$. Hat man nun den Wert von $\varphi$ erhalten; so lässt sich die Gleichung $X=0$ nach der Methode für eine Primzahl behandeln. Für

$$
n=2,3,4,5,6 \ldots
$$

hängt Lagrange's Methode von der Auflösung einer Gleichung rom Grade

$$
\rho=1,2,3,6,10 \ldots
$$

ab. Der Grad der Lagrange'schen Resolvente ist also stets. höher als der Grad der vorgelegten Gleichung, sobald $n>4$ ist. Trotzdem ist diese Methode ein bedeutender Fortschritt, denn die älteren Methoden führten auf eine Gleichung vom Grade $(n-1) !-$ so sind beispielsweise die Resolventen von Tschirnhaus, Euler und Bézout für die allgemeine Gleichung 5. Grades vom Grade 24, während die Lagrange'sche Resolvente nur vom Grade 6 ist.

Um die Coefficienten der Resolvente

$$
\theta^{4}-\mathrm{T} \theta^{3}+\mathrm{U}^{2}-\mathrm{X} \theta+\mathrm{r}=0
$$

zu berechnen, nahm Lagrange den Coefficienten T.

Er findet, dass

$$
\begin{aligned}
& \mathrm{T}=50 z-4 a_{1}^{5}+25\left(a_{1}^{3} a_{2}-a_{1}^{2} a_{3}+a_{1}\left(a_{4}-a_{2}^{2}\right)+a_{2} a_{3}-25 a_{5}\right) \\
& \text { ist, wo } \\
& \frac{6 !}{3 !(2 !)^{3}}=\frac{4.5 \cdot 6}{8}
\end{aligned}
$$




$$
\begin{gathered}
z=2\left\{x_{0}^{3}\left(x_{1} x_{4}+x_{2} x_{4}\right)+x_{1}^{3}\left(x_{0} x_{2}+x_{3} x_{4}\right)+x_{2}^{3}\left(x_{1} x_{3}+x_{0} x_{4}\right)+\right. \\
\left.+x_{3}^{3}\left(x_{2} x_{4}+x_{0} x_{1}\right)+x_{4}^{2}\left(x_{0} x_{3}+x_{1} x_{2}\right)\right\}+ \\
+3\left\{x_{0}\left(x_{1}^{2} x_{4}^{2}+x_{2}^{2} x_{3}^{2}\right)+x_{1}\left(x_{0}^{2} x_{2}^{2}+x_{3}^{2} x_{4}^{2}\right)+x_{2}\left(x_{1}^{2} x_{3}^{2}+x_{0}^{2} x_{4}^{2}\right)+\right. \\
\left.+x_{3}\left(x_{2}^{2} x_{4}^{2}+x_{0}^{2} x_{1}^{2}\right)+x_{4}\left(x_{0}^{2} x_{3}^{2}+x_{1}^{2} x_{2}^{2}\right)\right\}
\end{gathered}
$$

ist. Diese resolvierende Function hat ein gewisses historisches Interesse; denn Malfatti, Jacobi u. A. gebrauchten andere 6 wertige Functionen.

Da die Berechnung der Coefficienten der Gleichung in $z$ sehr mühsam ist, gibt Lagrange nur einen an, nämlich den des zweiten Gliedes. Übrigens scheint ihm diese Berechnung beinahe nutzlos, da sie "outre qu'il exigerait des calculs très longs, ne saurait d'ailleurs jeter aucune lumière sur la résolution des équations du cinquième degré ; car comme la reduite en $z$ est du sixième degré, elle ne sera pas resoluble à moins qu'elle ne puisse s'abaisser à un degré inférieur au cinquième; or c'est ce qui ne me parait guère possible d'après la forme des racines $z^{\prime}, z^{\prime \prime}$, . . . de cette équation".

An einer anderen wichtigen Stelle berührt Lagrange die Frage nach der Möglichkeit einer algebraischen Auflösung von Gleichungen höheren Grades. "Il semble," sagt er, "done qu'on pourrait conclure de là par induction que toute équation de quelque degré qu'elle soit, sera aussi resoluble à l'aide d'une reduite dont les racines soient representées par la même formule

$$
x_{0}+\alpha x_{1}+\alpha^{2} x_{2}+\ldots .+\alpha^{n-1} x_{n-1}
$$

Mais d'aprés ce que nous avons démontré . . . on a, ce semble, lieu de se convaincre d'avance que cette conclusion se trouvera en défaut dès le cinquième degré; d'où il s'ensuit que, si lá résolution algébrique des équation des degrès supérieurs au quatrième n'est pas impossible, elle doit dependre de quelques fonctions des racines, différentes de la précédente."

Schlieflich sei noch bemerkt, dass Lagrange seine Untersuchungen durch die Entdeckung einiger fundamentaler Sätze der Substitutionstheorie erleichterte und wesentlich förderte. So theilte er durch seine Definition von ähnlichen Functionen alle rationalen Functionen der Wurzeln in Classen ein; er zeigte sodann, dass ähnliche Functionen stets einer Gleichung von demselben Grade genügen, welcher ein Theiler von $n$ ! ist. Weiter bewies er, dass ähnliche Functionen rationale Functionen von einander sind, woraus folgt, dass jede rationale Function der Wurzeln durch eine $n$ ! wertige Function rational ausdrückbar ist. Dieser Satz scheint ihm "un des plus importants de la theorie des equations"; und 
hierin hat er Recht, denn Galois benutzte denselben als Grundlage seiner Theorie.

Endlich will ich noch herrorheben, dass Lagrange nur rationale Functionen der Wurzeln in den Kreis seiner Untersuchungen zieht und somit A bel's großen Satz über die rationale Ausdrückbarkeit der Wurzeln einer algebraisch auflösbaren Gleichung stillschweigend voraussetzt.

\section{Vandermonde.}

Vandermonde ${ }^{1}$ ) sucht, wie Euler und Bézout, die nothwendige Form einer Wurzel einer Gleichung; jedoch setzt er nicht, wie diese, voraus, dass die Auflösung eine algebraische sei, sondern frägt ausdrücklich, was die wesentliche Bedingung dafür sei, dass eine Gleichung eine algebraische Auflösung zulässt. Er findet, dass diese Bedingung in der Möglichkeit liegt, "de trouver une fonction de la somme des racines de leur produits deux-a-deux, de la somme de leurs produits trois-a-trois etc., qui soit indifferement l'une quelconque de ces racines". Die algebraische Auflösung einer Gleichung zerfällt also von selbst in drei Theile:

$1^{0}$ Trouver une fonction des racines de laquelle on puisse dire dans une certaine sens qu'elle égale telle de ces racines que l'on voudra.

$2^{0}$ Mettre cette fonction sous une forme telle qu'il soit de plus indifférent d'y echanger les racines entre elle.

$3^{0} \mathrm{Y}$ substituer les valeurs en somme de ces racines, somme de leurs produits deux-a-deux etc.

Eine Function von der in $1^{0}$ verlangten Art ist

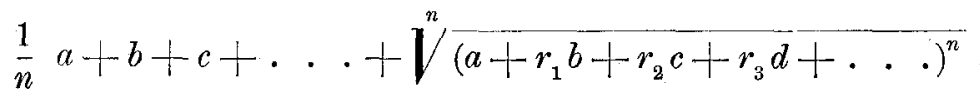

$$
\begin{aligned}
& +\sqrt{\left(a+r_{1}^{2} b+r_{2}^{2} c+r_{3}^{2} d+\ldots\right)^{n}} \\
& \left.+\sqrt{\left(a+r_{1}^{n-1} b+r_{2}^{n-1} c+r_{3}^{n-1} d+\cdot\right)^{n}}\right),
\end{aligned}
$$

wo $a, b, c, d, \ldots$ die Wurzeln der gegebenen Gleichung und $r_{1}, r_{2}, r_{3} . .$. die $n^{\text {ten }}$ Einheitswurzeln sind.

Vandermonde's Methode mag durch ein Beispiel erläutert werden. Für $n=3$ ist die Function

$$
\frac{1}{3}\left(a+b+c+\left(a+r_{1} b+r_{2} c\right)+\left(a+r_{2} b+r_{1} c\right)\right)=a
$$

1) Mémoire sur la résolution des équations. Hist. Acad. de Paris 1771. 
oder

$$
\frac{1}{3}\left(a+b+c+r_{1}\left(a+r_{1} b+r_{2} c\right)+r_{2}\left(a+r_{2} b+r_{1} c\right)\right)=c
$$

oder

$$
\frac{1}{3}\left(a+b+c+r_{2}\left(a+r_{1} b+r_{2} c\right)+r_{1}\left(a+r_{2} b+r_{1} c\right)\right)=b .
$$

Die Bedingung $1^{0}$ ist somit erfüllt. Der Bedingung $2^{n}$ kann ebenfalls genügt werden, denn wir haben:

$$
\frac{1}{3}[a+b+c+
$$

$\sqrt{\left(a^{3}+b^{3}+c^{3}\right)-\frac{3}{2}\left(a^{2} b+b^{2} c+c^{2} a+a^{3} c+b^{2} a+c^{2} b\right)+6 a b c+\frac{3}{2} \sqrt{-3} \sqrt{((a-b)(a-c)(b-c))^{2}}}$

$-\sqrt{\left.\left(a^{3}+b^{3}+c^{3}\right)-\frac{3}{2}\left(a^{3} b+b^{2} c+c^{2} a+a^{3} c+b^{3} a+c^{7} b\right)+6 a b c-\frac{3 \sqrt{-3}}{2} \sqrt{((a-b)(a-c)(b-c))^{2}}\right]}$

Die dritte Bedingung ist nun leicht zu erfüllen.

Nachdem Vandermonde die Gleichungen zweiten, dritten und vierten Grades erledigt hatte, suchte er seine Methode auf die Gleichung fünften Grades anzuwenden. Die Hauptschwierigkeit lag darin, der Bedingung $2^{\circ}$ zu genügen. Obschon seine Mühe ohne Erfolg war, ist es interessant, zu bemerken, dass Vandermonde rationale Resolventen vom fünften und sechsten Grade der allgemeinen Gleichung fünften Grades, sowie Resolventen vom zehnten und fünfzehnten Grade der allgemeinen Gleichung sechsten Grades entdeckte. Seine Resultate stimmten nicht nur mit den von Lagrange gefundenen in dieser Beziehung überein, sondern die Form 1) ist mit Lagrange's Form 3) geradezu identisch. Vandermonde machte noch die wichtige Bemerkung, dass er trotz allen Suchens keine Resolventen vom dritten und vierten Grade für die allgemeine Gleichung fünften Grades finden konnte und dass er die Überzengung gewonnen habe, dass solche Resolventen überhaupt nicht existieren. Schließlich mag hervorgehoben werden, dass. Vandermonde bemerkte, dass seine Methode eine Vereinfachung zulässt, wenn die gegebene Gleichung eine specielle ist. Als Beispiel führt er die Kreistheilungsgleichung

$$
x^{11}-1=0
$$

an, welche auf

$$
x^{5}-x^{4}-4 x^{3}+3 x^{2}+3 x-1=0
$$


zurückgeführt werden kann. Keine der bis dahin bekannten Methoden ist imstande, diese Gleichung zu lösen, während dies nach seiner Methode möglich ist.

\section{Malfatti.}

In Gegensatz zu Lagrange und Vandermonde, bedient sich Malfatti ${ }^{1}$ ) der Eliminationsmethoden von Euler und Bézout. Mit diesen letzten nimmt er folgende Form der Wurzeln an:

$$
\begin{aligned}
& x_{1}+\omega m+\omega^{2} p+\omega^{3} q+\omega^{4} n=0 \\
& x_{2}+\omega^{2} m+\omega^{4} p+\omega^{6} q+\omega^{8} n=0 \\
& x_{3}+\omega^{3} m+\omega^{6} p+\omega^{9} q+\omega^{12} n=0 \\
& x_{1}+\omega^{4} m+\omega^{8} p+\omega^{12} q+\omega^{16} n=0 \\
& x_{0}+\omega^{5} m+\omega^{10} p+\omega^{15} q+\omega^{20} n=0
\end{aligned} \quad\left(\omega^{5}-1=0\right)
$$

und findet durch eine sehr geschickt geführte Elimination eine Resolvente vom 6. Grade in $v=m n p q$. Malfatti war somit der erste, der die Rechnung wirklich zu Ende führte, und er fand keinen Nachahmer, bis Cockle, Harley und Cayley beinahe ein Jahrhundert später sich mit dieser Resolvente beschäftigten, ohne jedoch Kenntnis von Malfatti's Resultaten gehabt zu haben.

Die Gleichungen (1) geben

$$
\begin{aligned}
& \text { (2) }-5\left(m^{3} p+n^{3} q+m q^{3}+n p^{3}-m^{2} n^{2}+m n p q-p^{2} q^{2}\right) x+ \\
& +m^{5}+n^{5}+p^{5}+q^{5}+5(m n-p q)\left(m p^{2}+n q^{2}-m^{2} q-n^{2} p\right)=0 .
\end{aligned}
$$

Malfatti setzt

$$
\begin{array}{rl}
m n=y & p q=u \\
m^{2} q+n^{2} p=r \quad & m p^{2}+n q^{2}=t \\
m^{3} p+n^{3} q=\rho & m q^{3}+n p^{3}=\tau \\
\sqrt{r^{2}} \overline{t^{2}-4 r^{2} u^{2} y-4 t^{2} u y^{2}+16 u^{3} y^{3}}=u,
\end{array}
$$

und erhält aus (3) und (4)

$$
\begin{aligned}
& \rho u=r t-y \tau \\
& \rho \tau=r^{2} u+t^{2} y-4 u^{2} y^{2}
\end{aligned}
$$

1) De aequationibus quadrato-cubicis, disquisitio analytica. Atti dell' Accademia dei Fisiocritici di Siena 1771. 
und hieraus

$$
\begin{aligned}
& 2 p u=r t+\mu \\
& 2 \tau y=r t-\mu .
\end{aligned}
$$

Man erhält weiters

$$
\begin{aligned}
& \rho r=\left(m^{5}+n^{5}\right) u+y^{2} t \\
& \tau t=\left(p^{5}+q^{5}\right) y+u^{2} r .
\end{aligned}
$$

Die Elimination von $p$ aus (9) vermittelst (7) gibt

$$
m^{5}+n^{5}=\frac{(r t+\mu) r-2 t u y^{2}}{2 u^{2}}
$$

ähnlich ergibt die Elimination von $\tau$ aus (10) vermittelst (8)

$$
p^{5}+q^{5}=\frac{(r t-\mu) t-2 u^{2} r y}{2 y^{2}} .
$$

Hat die allgemeine Gleichung 5. Grades die Form

$$
x^{5}-5 a x^{3}+5 b x^{2}+5 c x+d=0,
$$

so erhalten wir durch Vergleichung mit (2)

$$
\begin{gathered}
y+u=a \\
r+t=b \\
p+\tau-y^{2}+y u-u^{2}=-c \\
m^{5}+n^{5}+p^{5}+q^{5}+5(y-u)(t-r)=d .
\end{gathered}
$$

Malfatti schreibt (16) und (17) in der Form $(17 \mathrm{a})-\frac{r^{2} t y^{2}+r t^{2} u^{2}-2 t u y^{4}-2 r u^{4} y+\left(r y^{2}-t u^{2}\right) \mu}{2 u^{2} y^{2}}+5(y-u)(t-r)=d$. und erhält aus (16a)

$$
\mu=\frac{2 c u y+r t(y+u)-2\left(y^{2}-u y+u^{2}\right) u y}{u-y} .
$$

Setzt man in diese Relation den Wert von $t$ aus (15) ein, und quadriert, so entsteht die Gleishung 


$$
\begin{aligned}
& r^{4}-2 b r^{3}+\left(2 y^{3}+2 u^{3}-a u y+b^{2}-a c\right) r^{2}- \\
& -\left(y^{3}+u^{3}+2 y(y-u)^{2}-a c\right) b r+ \\
& +b^{2} y(y-u)^{2}-6 y^{2} u^{2}\left(y^{2}+u^{2}\right)+11 y^{3} u^{3}+y u\left(y^{4}+u^{4}\right)+ \\
& \quad+c^{2} y u-2 c y u\left(y^{2}+u^{2}\right)+2 c y^{2} u^{2}=0
\end{aligned}
$$

welche Malfatti in der Gestalt

$$
\begin{aligned}
& r^{4}-2 b r^{3}+\left(2 y^{3}-y^{2} u-y u^{2}+2 u^{3}-c y-c u+b^{2}\right) r^{2}+ \\
& +-\left(-3 y^{3}+4 y^{2} u-2 y u^{2}-u^{3}+c y+c u\right) b r+ \\
& +y^{5} u-6 y^{4} u^{2}+11 y^{3} u^{3}-6 y^{2} u^{4}+y u^{5}-2 c y^{3} u-2 c y^{2} u^{2}- \\
& -2 c y u^{3}-c^{2} y u+b^{2} y^{3}-2 b^{2} y^{2} u+b^{2} y u^{2}=0
\end{aligned}
$$

schreibt. Setzt man den Wert von $\mu$ in $(17$ a) ein, so verwandelt sich diese Relation in

$$
\begin{gathered}
(y+u) r^{3}-(b y+2 b u) r^{2}+\left(2 y^{4}-12 y^{3} u+22 y^{2} u^{2}-12 y u^{3}+\right. \\
\left.(20)+2 u^{4}+b^{2} u-c y^{2}-c u^{2}\right) r+\left(-b y^{4}+6 b y^{3} u-11 b y^{2} u^{2}+\right. \\
\left.+6 b y u^{3}-b u^{4}+b c u^{2}-d y^{2} u+d y u^{2}\right)=0
\end{gathered}
$$

und eliminiert man endlich $r$ aus den letzten zwei Gleichungen, so erhält man eine Gleichung 6. Grades in $v=u y=m n p q$, welche, wenn man mit Malfatti

$$
25 u y=z+5 a^{2}-\frac{5 c}{3}
$$

setzt, die Gestalt

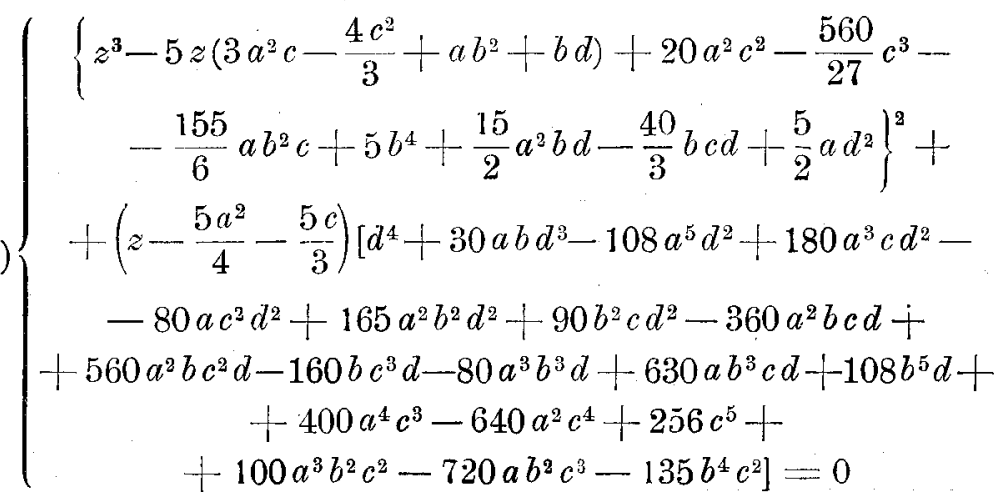

annimmt.

Diese Gleichung kann Malfatti in ihrer allgemeinen Form nicht aufösen; "non ideo particulares casus negligendos censeo, cum quibus haec resolutio succedit, praesertim cum mea methodus 
et omnibus quae hactenus resolutae sunt quinti gradus aequationibus sufficiat, ac ad plures alias se extendat quas Geometrae ad hane usque diem, veluti resolutionem Analyticam respuentes deseruerunt".

In der That entdeckt er, ohne es zu ahnen, jeden möglichen Fall der Aufösung, denn er zeigt, dass die allgemeine Gleichung vom 5. Grade stets auflösbar ist, sobald die Resolvente (22) einen rationalen Factor besitzt. Dass diese Bedingung der Auflösbarkeit nicht nur hinreichend, sondern auch nothwendig ist, wurde von Luther $\left.{ }^{1}\right) 1847$ bewiesen.

Die Malfattische Resolvente (22) ist mit der Lagrange'schen eng verwandt. In der That geben die Gleichungen (1)

$$
\left\{\begin{array}{l}
-5 m=x_{0}+\omega^{4} x_{1}+\omega^{3} x_{2}+\omega^{2} x_{3}+\omega x_{4} \\
-5 p=x_{0}+\omega^{3} x_{1}+\omega x_{2}+\omega^{4} x_{3}+\omega^{2} x_{4} \\
-5 q=x_{0}+\omega^{2} x_{1}+\omega^{4} x_{2}+\omega x_{3}+\omega^{3} x_{4} \\
-5 n=x_{0}+\omega x_{1}+\omega^{2} x_{2}+\omega^{3} x_{3}+\omega^{4} x_{4}
\end{array}\right.
$$

Es ist somit $5^{4} m p q n=5^{4} v=\sqrt[3]{\Upsilon}$, wo $\Upsilon$ das absolute Glied der Lagrange'schen Resolvente 4. Grades

$$
\theta^{4}-\mathrm{T} \theta^{3}+\mathrm{U} \theta^{2}-\mathrm{X} \theta+\mathrm{r}=0
$$

ist. Die Gleichungen (23) geben:

$$
\begin{aligned}
& 5^{2} m n=\sum x^{2}+\left(\omega+\omega^{4}\right) t_{1}+\left(\omega^{2}+\omega^{3}\right) t_{2} \\
& 5^{2} p q=\sum x^{2}+\left(\omega^{2}+\omega^{3}\right) t_{1}+\left(\omega+\omega^{4}\right) t_{2},
\end{aligned}
$$

wo $t_{1}=(0123)=x_{0} x_{1}+x_{1} x_{2}+x_{2} x_{3}+x_{3} x_{4}+x_{4} x_{0}, t_{2}=(02413)$ ist, so dass also $v=m n p q$ lediglich von der 6 -wertigen Function $t_{1} t_{2}=\tau$ abhängt. Eine einfache Rechnung gibt

$$
z=-\frac{1}{15}(20 c+3 \varphi)
$$

wo

$$
\varphi=\sum_{0}^{4} x_{k}^{2}\left(x_{k+1} x_{k+4}+x_{k+2} x_{k+3}\right)
$$

ist. Lagrange scheint nicht bemerkt zu haben, dass die fünfte Wurzel aus $\mathrm{r}$ zu einer einfacheren Resolvente als der Coefficient $T$ führt. Wir werden sehen, dass Jacobi eine neue Resolvente entdeckte, indem er die sechswertige Function $y=t_{1}-t_{2}$ gebrauchte.

1) E. Luther. De criteriis quibus cognoscatur an aequatio quinti gradus irreductibilis algebraice resolvi possit. Crelle, Bd 34 . 


\section{Ruffini. 1)}

Nach dem Jahre 1771 beginnt eine neue Epoche in der Geschichte unserer Gleichung. Der durch Tschirnhaus und Euler eröffnete Weg ist durch eine unüberwindliche Schwierigkeit gesperrt; die Reduction der Resolvente vom vierundzwanzigsten Grade auf eine solche rom sechsten war der letzte mögliche Schritt in dieser Richtung. Ein durch die erfolglose Arbeit eines Jahrhunderts berechtigter Zweifel bemächtigt sich nunmehr vieler Mathematiker.

Schon Lagrange, entmuthigt durch die ungeheueren Berechnungen, welche eine weitere Untersuchung verlangen würde, betrachtet "le succés fort douteux", während Vandermonde nur an der Existenz einer Resolvente dritten oder vierten Grades zweifelte. Diese Bedenken wurden noch klarer in der Dissertation des 22jährigen Gau ß (1799) ausgesprochen:

". - post tot tantorum geometrartim labores perexiguam spem superesse, ad resolutionem generalem aequationum algebraicarum unquam perveniendi, ita ut magis magisque verisimile fiat, talem resolutionem omnino esse impossibilem et contradictoriam. Hoc eo minus paradoxum videri debet, qu um id quod vulgo resolutio aequationis dicitur, proprie nihil aliud sit quam ipsius reductio ad aequationes puras. Nam aequationum purarum solutio hine non docetur sed supponitur, et si radicem aequationis $x^{m}=H$ per $\sqrt[m]{\mathrm{H}}$ exprimis, illam neutiquam solvisti neque plus fecisti, quam si ad denotandam radicem aequationis $x^{n}+A x^{n-1}+$ etc. $=0$ signum aliquod excogitares, radicemque hine aequalem poneres. Verum est aequationes puras propter facilitatem ipsarum radices per approximationem inveniendi, et propter nexum elegantem, quem omnes radices inter se habent, prae omnibus reliquis multum praestare, adeoque neutiquam vituperandum esse, quod analystae harum radicis per signum peculiare denotaverunt; attamen ex eo, quod hoc signum perinde ut signa arithmetica addi-

1) Della soluzione delle equazioni algebraiche determinate particolari di grado superiore al quarto. Mem. Soc. Ital. Bd. 9.

Della insolubilità delle equazioni algebraiche generali di grado superiore al quarto. Mem. Soc. Ital. Bd, 10.

Riposta di P. R. ai dubji propostogli dal socio G-Fr. M. sopra la insolubilità algebraica dell' equazioni di grado superiore al quarto. Mem. Soc. Ital. Bd. 12. Riflessioni intorno al metodo proposto dal consocio Malfatti per la soluzione della equazioni di $5^{0}$ grado. Mem. Soc. Ital. Bd. 12.

Della insolubilità delle equazioni algebraiche generali di grado superiore al $4^{0}$ qualunque metodo si adoperi, algebraico esso o transcendentale. Mem. Inst. Naz. Ital. Bd. 1.

Riflessioni intorno alla soluzione delle equazioni algebraiche generali. Modena 1813.

Intorno al metodo generale proposto dal sig Hoëné Wronski onde risolvere le equazioni di tutti $i$ gradi. Mem. Soc. Ital. $t 18,1820$.

Eine eingehende Würdigung der Arbeiten Ruffini's hat Herr $\mathbf{B}$ urkhard im Supplement zum 37. Jahrgange der Zoitschrift für Mathematik und Physik veröffentlicht; wir können uns daher hier sehr kurz fassen, indem wir den Leser auf diese ausgezeichnete Darstellung verweisen. 
tionis, subtractionis, multiplicationis, divisionis et evectionis ad dignitatem sub nomine expressionum analyticarum complexi sunt, minime sequitur, cuiusvis aequationis radicem per illas exhiberi posse. Seu, missis verbis, sine ratione sufficienti supponitur, cuiusvis aequationis solutionem ad solutionem aequationum purarum reduci posse. Forsan non ita difficile foret, impossibilitatem iam pro quinto gradu omni rigore demonstrare, de qua re alio loco disquisitiones meas fusius proponam".

Die Zeit war also reif für eine neue Epoche, die mit der Veröffentlichung von Ruffin i's Werk ${ }^{1}$ ) "Teoria generale delle Equazioni in cui si dimostra impossibile la scluzione algebraica delle equazioni generali di grade superiore al quarto" beginnt.

Ruffini bedient sich der Methoden und Sätze von Lagrange, dessen großes Verdienst er mit folgenden warmen Worten anerkennt: "L'immortale de la Grange, con le sublimi sue Riflessioni intorno alle Equazioni inserite negli Atti dell' Accademia di Berlino a somministrato il fondamento alla mia dimostrazione".

Diesem Beweise in seiner "Teoria" folgten fünf andere. Ich wähle den letzten von diesen, nämlich den vom Jahre 1813, welcher, obschon im Ganzen weniger interessant als die anderen, doch in mancher Beziehung als die Frucht seiner reifsten Überlegung über diesen spröden Gegenstand gelten kann.

Es sei

$$
x^{n}+a x^{n-1}+b x^{n-2}+c x^{n-3}+\ldots .=0
$$

eine allgemeine Gleichung $n^{\text {ten }}$ Grades. Ruffin i zeigt, dass jede algebraische Function der Größjen $a, b, c$. . a auf folgende Weise erhalten werden kann:

Es sei $P$ eine rationale Function von $a, b, c . .$. ; alsdann erhalten wir eine erste Irrationalität $Q$, indem wir eine $p^{\text {te }}$ Wurzel aus $P$ ziehen, wo $p$ eine Primzahl vorstellt, so dass

$$
Q^{p}=P
$$

ist.

Es sei ferner $F_{1}$ eine rationale Function von $P$ und $Q$; eine zweite Irrationalität wird dann durch die Gleichung

definiert $\mathfrak{u}$. s. f.

$$
R^{q}=F_{1}
$$

Ora qualunque siasi il metodo, per cui si voglia potersi ottenere la soluzione algebraica essatta della data equazione (1) esso non può mai che condurre alla determinazione di certe functioni algebraiche finite de' coifficienti, $a, b, c$ le quali sostituite in luogo della $x$

1) Bologna 1799 . 
facciano verificàre l'equazione medesima, e, cosi ne costituiscono le diverse radici. Dunque ogniqualvolta la (1) ammettesse soluzione algebraiche essatta dovrebbe sempre potersi determinare un espressione della forma

$$
F(P, Q, R, S . . .)
$$

uguale ad una della radici $x_{1} x_{2} \ldots$

Bezüglich der Beschaffenheit von $F$ behauptet $R$ uffini, dass dieselbe irrational sein müsse, denn sonst würde (1) eine rationale Wurzel besitzen. Weiters muss die Function $F$ derart beschaffen sein, dass, wenn wir darin $a, b, c, \ldots$ durch ihre Werte $-\left(x_{1}+x_{2}+\ldots\right), x_{1} x_{2}+x_{1} x_{3}+\ldots$ etc. ersetzen and die angedeuteten Operationen austühren, dieselbe sich auf eine der Größjen $x_{m}$ reduciert

Es ist sehr interessant, zu sehen, wie $R u f f i n i$ seine Behauptung begründet. "Difatti eseguite le accennate sostituzioni e riduzioni, se si volesse che essa (A) divenuta funzione delle $x_{1}, x_{2} \ldots$ rimanesse irrazionale col non potere scompire mediante lattuali estrazione dell' radici tutti i vincoli radicali; allora dovendo questa (A) per la indeterminazione delle $x_{1} x_{2} \ldots$ essere non solamente uguale ma identica con la $x_{m}$ averemmo un' espressione razionale, cioè $x_{m}$ identica con un' irrazionale, il che e un' assurdo. Nel modo stesso si vede dovere la (A) diventare funzione delle $x_{1}, x_{2} \ldots$ intera; perchè altramente si avrebbe la espressione intera $x_{m}$ identica con un espressione fratta, il che è nuovamente un assurdo. Finalmento nella equazione $x_{m}=F$ il secondo membro dela quale abbiamo demostrato non potere essere che una funzione razionale ed intera della $x_{1} x_{2} \ldots$ siccome il primo membro $\dot{a}$ formato del solo termine $x_{m}$ dovranno nel membro secondo (A) per l'indicata indenticità e pei noti principi, eliminarsi spontaneamente tutti $i$ termini e rimanervi il solo $x_{m}{ }^{\prime \prime}$.

Es folgt nun der wichtige Schluss: „Dunque dopo le accennate sostituzioni e reduzioni le funzioni dei coefficienti $a, b, c \ldots$ che nella (A) si contengono sotto vincoli radicali dovrannodiventare tante funzioni delle $x_{1}, x_{2}, \ldots$ che siano potenze essatte in modo che si potranno da' loro estrarre attualmente le radici da' vincoli medesimi".

Für die Gleichungen zweiten, dritten und vierten Grades zeigt Ruffini, dass dies wirklich der Fall sei.

Beispielsweise ist

$$
\sqrt[3]{-\frac{c}{2}+\sqrt{\frac{c^{2}}{4}+\frac{b^{3}}{27}}}-\sqrt[3]{-\frac{c}{2}-\sqrt{\frac{c^{2}}{4}+\frac{b^{3}}{27}}}
$$

eine Wurzel der Gleichung

$$
x^{3}+b x+c=0 .
$$


Die Größe unter dem Quadratwurzelzeichen ist gleich

$$
-\frac{1}{4.27}\left(2 x_{2}^{3}+3 x_{2}^{2} x_{3}-3 x_{2} x_{3}^{2}-2 x_{2}^{3}\right)^{2}
$$

also, abgesehen von einem numerischen Factor, ein vollkommenes Quadrat; die zwei Größen unter Cubikwurzelzeichen sind gleich

$$
\frac{1}{27}\left(x_{1}+\beta^{2} x_{2}+\beta x_{3}\right)^{3} \text { bezw. } \frac{1}{27}\left(x_{1}+\beta^{2} x_{3}+\beta x_{2}\right)^{3}
$$

und somit vollkommene Cuben, und endlich ist die Summe ihrer Cubikwurzeln gleich $x_{1}, x_{2}$ oder $x_{3}$, je rach dem Werte, den wir den Wurzeln beilegen. Wir weisen im Vorübergehen auf die $\ddot{\Lambda} \mathrm{hn}$ lichkeit dieser Betrachtungen mit denen von Vandermonde hin.

Ruffini hebt noch eine wichtige Eigenschaft der Function $F$ hervor, falls dieselbe einer Gleichung von niedrigerem als dem fünften Grade genügt. Er sagt:

"Riflettarsi finalmente che le attuali estrazioni delle radici quelle sono nella soluzione algebrica delle Equazioni che possono far perdere alle $x_{1}, x_{2}, \ldots$ quello stato simmetrico ossia quel rapporto uguale di posizione, che esse avevano nei coefficienti $a, b, c, \ldots$; di piu le estrazioni medesime, e le altre operazioni algebriche practicate sulle funzioni delle $x_{1}, x_{2}, \ldots$ che ei risultano possono agire sopra le funzioni medesime in modo che esse le quali prima delle indicata operazioni conservarano il proprio valore sotto certe permutazioni fra le $x_{1}, x_{2}, \ldots$ non lo conservano piu in seguito. Da cio è che nelle equazioni de gradi $2^{0}, 3^{\prime \prime}, 4^{0}$ se ne può ottenere la soluzione".

So bleibt z. B. die Gröbe

$$
\frac{c^{2}}{4}+\frac{b^{3}}{27}=-\frac{1}{4.27} f\left(x_{1}, x_{2}, x_{3}\right)^{2}
$$

nach jeder Vertauschung von $x_{1}, x_{2}, x_{3}$ unverändert, während

$$
\sqrt{\frac{c^{2}}{4}+\frac{b^{3}}{27}}=\frac{\sqrt{-3}}{2.9} f\left(x_{1}, x_{2}, x_{3}\right)
$$

bei jeder Vertauschung zweier Elemente $x_{\alpha}, x_{\beta}$ das Zeichen ändert. Ähnliche Bemerkungen gelten für die Grölben unter dem Cubikwurzelzeichen vor und nach der Wurzelausziehung.

Nach diesen Vorbemerkungen ist Ruffini imstande, den gruppentheoretischen Theil seines Beweises anzutreten.

Wir setzen

$$
\begin{aligned}
& y=Q=f\left(x_{1}, x_{2}, x_{3}, x_{4}, x_{3} \ldots\right)=f(12345) \\
& y^{p}=P=F\left(x_{1}, x_{2} \ldots \ldots \ldots \ldots\right)=F(12345)
\end{aligned}
$$


nnd es seien $y_{1}, y_{2}, y_{3}, y_{4}, y_{5}$ die Werte von $y$ nach Anwendung

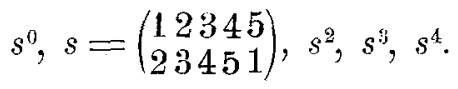

Wendet man nun $s, s^{2} \ldots$ anf die Identität

$$
\{f(12345)\}^{p}=y_{1}^{p}=F(12345)
$$

an, so erhält man

$$
y_{1}^{p}=y_{2}^{p}=\ldots=y_{5}^{p}=F
$$

Es sind somit $y_{1}, y_{2}, \ldots$ Wurzeln der Gleichung (2) und

$$
y_{2}=3 y_{1}
$$

wo $\beta$ eine primitive $p^{\text {te }}$ Einheitswurzel ist.

Wendet $\operatorname{man} s, s^{2}, \ldots$ auf diese Gleichung an, so ergibt sich

$$
y_{2}=\beta y_{1}, \quad y_{3}=\beta^{2} y_{1}, \quad y_{4}=\beta^{3} y_{1}, \quad y_{5}=\beta^{4} y_{1}, \quad y_{1}=\beta^{5} y_{1},
$$

und daher $\beta^{5}=1$.

Die Gleichung: (2) lässt ferner auch die Substitutionen

$$
\sigma=\left(\begin{array}{lll}
1 & 2 & 3 \\
2 & 3 & 1
\end{array}\right), \sigma^{2}
$$

zu. Setzt man nun

$$
y_{a}=f(23145), \quad y_{a+1}=f(31245),
$$

so entstehen die neuen Relationen

$$
y_{a}=\gamma y_{1}, \quad y_{a+1}=\gamma^{2} y_{1}, \quad y_{1}=\gamma^{3} y_{1},
$$

aus denen $\gamma^{3}=1$ folgt.

$W$ ir haben ferner

$$
f(23451)=y_{2}=\beta y_{1},
$$

welche nach Anwendung von $\sigma$ die Gleichung

liefert.

$$
f(31452)=\beta \gamma y_{1}
$$

Setzt man also

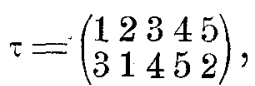


und wendet $\tau, \tau^{2}, \ldots$ auf diese Relation an, so ergibt sich:

$$
3^{5} \gamma^{5}=1
$$

somit $y=1$, also

$$
y_{1}=y_{a}=y_{a+1} \text {. }
$$

Setzt man ferner

$$
y_{c}=f(12453), \quad y_{c+1}=f(12534),
$$

so ist

$$
y_{e}=\grave{o} y_{1} \text { und } \delta^{3}=1
$$

Es besteht weiter die Relation

$$
f(23451)=\beta f(12345),
$$

welche nach Anwendung von

$$
\left(\begin{array}{lll}
3 & 4 & 5 \\
4 & 5 & 3
\end{array}\right)
$$

in

übergeht.

$$
f(24531)=3 \% y_{1}
$$

Nach Anwendung von

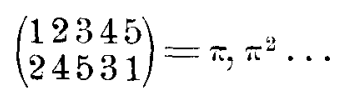

erhält man ferner die Relation

somit

$$
\beta^{5} \delta^{5}=1 \text {, oder } \delta=1 ;
$$

$$
y_{1}=y_{c}=y_{c+1} \text {. }
$$

Wir haben endlich nach Anwendung von $\sigma$ auf

$$
y_{1}=y_{a}=y_{c}
$$

die Relation

$$
y_{1}=y_{a}=y_{2}
$$

Da nun $y_{2}=\beta y_{1}$, so ist $\beta=1$, somit

$$
y_{1}=y_{3}=y_{5}=y_{4}=y_{5},
$$

Es folgt daraus, dass $Q$ bei den Substitutionen $s, s^{2}, \ldots$ unverändert bleibt. Dasselbe gilt somit auch für $R, S, \ldots$ und schließlich für $F$. 
Die Gleichung

$$
x_{m}=F(P, Q, R, \ldots)
$$

ist somit absurd, denn die rechte Seite bleibt bei gewissen Vertauschungen der Größen $x_{1}, x_{2}, \ldots$ unverändert, während die linke sich ändert.

\section{Abel.}

Während Abel noch auf dem Gymnasium zu Christiania war, glaubte er kurze Zeit im Besitze der algebraischen Auflösung der allgemeinen Gleichung fünften Grades zu sein und wollte eine Abhandlung über diesen Gegenstand der königl. Akademie der Wissenschaften zu Kopenhagen vorlegen. Er entdeckte jedoch sehr bald seinen Fehler, als man ihn bat, seine Rechnungen etwas detaillierter zu machen und dieselben auf numerische Beispiele anzuwenden. Bjerknes bemerkt hierüber in seinem ausgezeichneten "Vie d'Abel": Au lieu de faire comme Ja cobi et d'abandonner le dangereux problème sur la résolution duquel tant des hommes distingués avaient usé leurs forces, il continua avec acharnement la lutte sous se laisser effrayer par les conclusions du geomètre ${ }^{1}$ ) danois touchant la sterilité de ce sujet difficile. Après un labeur, ou pour mieux dire, un combat de plussieurs années il parvint enfin ̀̀ trouver l'explication de l'impénétrable mystère ", und im Jahre 1824 lässt er auf eigene Kosten die sehr knapp gefasste Abhandlung ${ }^{2}$ ) betitelt "Mémoire sur les équations algebriques ou l'on démontre l'impossibilité de la resolution de l'equation générale du cinquième degré" erscheinen. Diesem nicht ganz stichhältigen Beweise folgte zwei Jahre später ein zweiter, ${ }^{3}$ ) welcher in dem ersten Bande des Crelle'schen Journals veröffentlicht wurde. Es ist interessant zu bemerken, dass $\mathrm{Abel}$ schon in der ersten $\mathrm{Ab}$ handlung den schwachen Punkt des Ruffini'schen Beweises zu vermeiden wusste, indem er durch ausführliche Untersuchung das Postulat seines Vorgängers streng beweist. Dagegen fehlt Abel in dem substitutions-theoretischen Theil seines Beweises, welcher $\mathrm{R}$ uffini's Stärke bildet.

Ich will jetzt zeigen, wie Abel den algebraischen Theil seines großen Beweises erbrachte, und bemerke nebenbei, dass er weder bei der ersten, noch bei der zweiten Redaction desselben von der Existenz der Arbeiten Ruffini's Kenntnis hatte. Es sei $x_{1}, x_{2}, x_{3} \ldots$ eine endliche Anzahl von Größen, und $p^{\prime}, p^{\prime \prime}, \ldots$ rationale Functionen derselben. Ist $f(\alpha, \beta, \ldots \gamma)$ eine rationale

1) Prof. Degen.

2) Oeuvres Bd. I.

s) Démonstration de l'impossibilité etc. Oeuvres Bd. I. 
Function von $\alpha, \beta, \ldots, \gamma$ und sind $n^{\prime}, n^{\prime \prime}, \ldots$ beliebige Primzahlen, so nennt man

$$
p_{1}=f\left(x_{1}, x_{2}, \ldots \sqrt[n^{\prime}]{p^{\prime}}, \sqrt[n^{\prime \prime}]{p^{\prime \prime}} \ldots\right)
$$

eine algebraische Function erster Ordnung

$$
p_{2}=f\left(x_{1}, x_{2}, \ldots \sqrt{p^{\prime}} \ldots \sqrt{p_{1}^{\prime}} \ldots\right)
$$

eine algebraische Function zweiter Ordnung u. s. f. Eine algebraische Function $\mu^{\text {ter }}$ Ordnung kann man also durch

$$
v=f\left(r^{\prime}, r^{\prime \prime}, \ldots \sqrt[n]{p}\right)
$$

darstellen, wo $p$ eine Function $\mu-1^{\text {ter }}$ Ordnung ist, und $r^{\prime}, r^{\prime \prime} \ldots$ Functionen niedriger Ordnung sind; selbstverständlich darf $p^{\frac{1}{n}}$ in den $r$ nicht rational sein.

Wir können auch $v$ wie folgt schreiben:

$$
v=\frac{t_{0}+t_{1} p^{\frac{1}{n}}+\cdots+t_{m} p^{\frac{n}{n}}}{v_{0}+v_{1} p^{\frac{1}{n}}+\cdots+v_{m} p^{\frac{m^{\prime}}{n}}}=\frac{T}{V}
$$

wo $t, v$ rationale, ganze Functionen der $r$ sind. Es seien $V_{1}, V_{2} \ldots V_{n-1}$ die $n-1$ Werte, die $V$ annimmt, wenn man darin $p^{\frac{1}{n}}$ durch $\alpha p^{\frac{1}{n}}, \alpha^{2} p^{\frac{1}{n}} \ldots$ ersetzt, wo $\alpha$ eine primitive $n^{\text {te }}$ Einheitswurzel ist. Dann ist

$$
v=\frac{T V_{1} V_{2} \ldots V_{n-1}}{V V_{1} V_{2} \ldots V_{n-1}^{-}}=q_{0}+q_{1} p^{\frac{1}{n}}+q_{2} p^{\frac{2}{n}}+\ldots+q_{n-1} p^{\frac{n-1}{n}},
$$

wo $q_{0}, q_{1}, \ldots$ rational in $p$, und den $r$ sind, während $p^{\frac{1}{n}}$ es nicht ist.

Wir können $v$ stets auf die Form

$$
v=q_{0}^{\prime}+p_{1}^{\frac{1}{n}}+q_{2}^{\prime} p_{1}^{\frac{2}{n}}+\ldots+q_{n-1}^{\prime} p_{1}^{\frac{n-1}{n}}
$$

bringen, wo $p_{1}^{\frac{1}{n}}$ nicht rational in $p_{1}, q_{0}, \ldots$ ist. 
Es möge nun der Ausdruck

$$
y=q_{0}+p^{\frac{1}{n}}+q_{2} p^{\frac{2}{n}}+\ldots+q_{n-1} p^{\frac{n-1}{n}}
$$

einer algebraischen Gleichung:

$$
y^{r}+c_{1} y^{r-1}+\ldots+c_{r}=0
$$

genügen, welche nach Einsetzung des Wertes von $y$

$$
\alpha_{0}+a_{1} p^{\frac{1}{n}}+\ldots+\alpha_{n-1} p^{\frac{n-1}{n}}=0
$$

wird, wo $\alpha_{0}, \alpha_{1}, \ldots$ rational in $p, q_{0}, \ldots$ sind. A $\mathrm{Abel}$ beweist nun, dass

a)

$$
\alpha_{0}=0, \quad \alpha_{1}=0, \ldots, \alpha_{n-1}=0
$$

ist; denn sonst hätten die Gleichungen

$$
\begin{gathered}
z^{n}-p=0 \\
\alpha_{0}+\alpha_{1} z+\ldots+\alpha_{n-1} z^{n-1}=0
\end{gathered}
$$

gemeinsame Wurzeln, und $p^{\frac{1}{n}}$ wäre dann in $q_{0}, \ldots$ rational, was der Annahme widerspricht. Das Übrige ist sehr leicht zu folgern. In der Tbat, vermöge (a), genügen der Gleichung (1) auch die Werte von $y$, welche man erbält, wenn man $p^{\frac{1}{n}}$ durch $\alpha p^{\frac{1}{n}} \alpha^{2} p^{\frac{1}{n}}, \ldots$ ersetzt. Bezeichnet man dieselben mit $y_{1}, y_{2}, \ldots$, so ist

$$
\begin{aligned}
& y_{1}=q_{0}+p^{\frac{1}{n}}+q_{2} p^{\frac{2}{n}}+\ldots q_{n-1} p^{\frac{n-1}{n}} \\
& y_{2}=q_{0}+\alpha p^{\frac{1}{n}}+\alpha^{2} q_{2} p^{\frac{2}{n}}+\ldots \alpha^{n-1} q_{n-1} p^{\frac{n-1}{n}}
\end{aligned}
$$

und somit

$$
\begin{aligned}
& q_{0}=\frac{1}{n}\left(y_{1}+y_{2}+\ldots+y_{n}\right) \\
& p^{\frac{1}{n}}=\frac{1}{n}\left(y_{1}+\alpha^{n-1} y_{2}+\ldots+\alpha y_{n}\right)
\end{aligned}
$$


und es erhellt daraus, dass $p^{\frac{1}{n}}, q_{0}, \ldots$ rationale Functionen der Wurzeln der vorgelegten Gleichung (1) sind. Es sei nun

$$
x^{m}+a_{1} x^{m-1}+\ldots+a_{m}=0
$$

eine allgemeine algebraisch auflösbare Gleichung $m^{\text {ten }}$ Grades und

$$
x=s_{0}+v^{\frac{1}{n}}+\ldots+s_{n-1} v^{\frac{n-1}{n}} .
$$

Nach dem vorhergehenden sind $v, s_{0}, s_{2}, \ldots$ rational in den Wurzeln $x_{1}, x_{2}, \ldots, x_{m}$ der Gleichung (2).

Sind $v_{1}, v_{2}, \ldots, v_{n}$ die Werte, die $v$ annimmt, wenn $\operatorname{man} x_{1}, x_{2}, \ldots$ auf alle möglichen Weisen permutiert, so werden die Coefficienten derjenigen Gleichung, deren Wurzeln die $v_{1}, v_{2} \ldots$ sind, rationale Functionen der $a_{1}, a_{2}, \ldots$

Daher ist

$$
v=t_{0}+u^{\frac{1}{v}}+t_{2} u^{\frac{2}{v}}+\cdots+t_{v-1} u^{\frac{v-1}{v}}
$$

wo die Größen $u^{\frac{1}{v}}, t_{0}, \ldots$ rational in den $v_{1}, v_{2}, \ldots$ und somit in den $x_{1}, x_{2}, \ldots$ sind. Satz:

Auf diese Weise beweist $\mathrm{Abel}$ den außerordentlich wichtigen

"Wenn eine Gleichung algebraisch auflösbar ist, so kann man der Wurzel stets eine solche Form geben, dass sich alle algebraischen Functionen, aus welchen sie zusammengesetzt ist, durch rationale Functionen der Wurzeln der gegebenen Gleichung ausdrücken lassen."

Dieses Theorem wurde von Vandermonde in etwas anderer Form zuerst deutlich ausgesprochen; es bildet, wie wir schon angedeutet haben, die nicht völlig genügend bewiesene Grundlage des $R$ uffini'schen Beweises.

Bei dem substitutionentheoretischen Theil des Abel'schen Beweises wollen wir uns nicht aufhalten, da $R$ uffini's Betrachtungen sich weit einfacher anwenden lassen.

Interessant ist es jedoch, das Schicksal von $A$ bel's berïhmten Beweise zu verfolgen. Im Jahre 1832 erklärte die Prager Gesellschaft der Wissenschaften die Beweise von Ruffini und Abel für unbefriedigend und schrieb einen Preis für einen vollkommen befriedigenden Beweis der Unauflösbarkeit der Gleichungen von höherem als dem vierten Grade oder die Auffindung der Auflösung aus. Poin s ot betrachtete die Frage über die Auflösbarkeit der Gleichungen höheren Grades als noch unerledigt, und $\mathrm{Hamilton}$, der geniale Erfinder der Quaternionen, widmete dem Gegenstande eine lange 
Abhandlung in den "Transactions of the Royal Irish Society" des Jahres 1839, da ihn weder der algebraische noch der substitutionentheoretische Theil des Abel'schen Beweises befriedigte. Cockle bemerkt im "Philosophical Magazine" Bd. 7, Ser. IV, dass "the joint argument of $\mathrm{Abel}$ and Hamilton appears to have been assented to by Murphy but grave doubts respecting it have been thrown out by Dr. P e ac o ck"; Ivory und Jerrard werden daselbst als Anhänger für die Möglichkeit einer algebraischen Auflösung der Glejchung fünften Grades angeführt. Schließlich ist es eine bemerkenswerte Thatsache, dass wir in einem so vorzüglichen Handbuche, wie Serret's "Algèbre Supérieure" ist, nicht nur Abel's Classt cation algebraischer Functionen in Bezug auf "ordre", sondern auch in Bezug auf "degré" finden. Bezüglich des letzteren machte $\Delta$ bel einen Fehler, welcher, obschon durch ihn die Giltigkeit des Beweises nicht im geringsten beeinflusst wird, $\mathrm{Hamilton}$ veranlasste, zu bemerken: "it renders it difficult to judge of the validity of his subsequent reasoning ".

Erst nachdem die Kenntnis von Hermite's und Kronecker's Auflösung der allgemeinen Gleichung tünften Grades durch elliptische Functionen sich verbreitet hatte, sowie das Studium der Gruppentheorie allgemeiner wurde, verschwand der Glaube an eine algebraische Auflösung. A bel bemerkte ${ }^{1}$ ) selbst von seinem Beweise: „Je crois que la demonstration que j'ai donnée dans le premier cahier de ce journal ${ }^{2}$ ) ne laisse rien à désirer du côté de la rigueur; mais elle n'a pas toute la simplicité dont elle est susceptible ${ }^{u}$. Diese Vereinfachung, welche zu veröffentlichen Abel durch seinen frühzeitigen Tod verhindert war, wurde von $\mathrm{K}_{\mathrm{r}}$ on e $\mathrm{cker}^{3}$ ) in der Abhandlung "Vereinfachung des Abelsehen Beweises etc." geliefert.

Noch viele andere Ansprüche aber hat $\mathrm{Abel}$ auf unsere Bewunderung; denn derselbe geniale Blick, welcher das Geheimnis der Gleichung füntten Grades aufdeckte, erschloss auch die Theorie der elliptischen Functionen. Bald nach Abel fieng auch Jacobi an, die neue Theorie durch seine Untersuchungen zu bereichern, und es dauerte nicht lange, bis beide auf ganz verschiedenen Wegen $z u$ einer Classe von Gleichungen geführt wurden, die später eine sehr große Rolle spielen sollte, nämlich zu den Modulargleichungen.

Wir wollen hier kurz darlegen, wie man nach Abel und $\mathrm{Jacobi}$ zu diesen Gleichungen gelangt.

Beschäftigen wir uns zuerst mit $\mathrm{A}$ bel.

Nachdem dieser $s n n v$ in $s n v, c n v, d n v$ rational ausgedrïckt hat, tritt er das umgekehrte Problem an, nämlich $\operatorname{sn} \frac{v}{n}$ durch $\operatorname{sn} v$, cnv und $d n v$ auzudrücken.

1) Nachlass. Oeuvres Bd. II. Sur la résolution algébrique des Equations.

$\left.{ }^{2}\right)$ Journal der reinen und angewandten Mathematik.

3) Monatsberichte der königl, preuß. Akad. d. Wiss. 1879. 
Wir wollen der Einfachheit halber voraussetzen, $n$ sei eine ungerade Primzahl. Dann ist $\operatorname{sn} \frac{v}{n}$ Wurzel der Gleichung $n^{\text {2ten }}$ Grades

$$
F(x)=Q\left(x^{2}\right) \operatorname{sn} v-x P\left(x^{3}\right)=0 .
$$

Diese Gleichung ist, wie Abel zeigte, algebraisch auflösbar, wenn die Größen

$$
\begin{aligned}
y_{p, q}=s n\left(\frac{4 p K+4 q K^{\prime} i}{n}\right) \quad(p, q & =0,1,2 \ldots n-1 ; \\
p & =q=0 \text { ausgeschlossen })
\end{aligned}
$$

als bekannt angesehen werden. Diese Gröben sind Wurzeln der Gleichung $\left(n^{2}-1\right)^{\text {ten }}$ Grades

$$
P\left(y^{2}\right)=0,
$$

welche sich auf eine algebraisch autlösbare Gleichung $(n+1)^{\text {ten }}$ Grades zurückführen lässt.

In der That gehören die Gleichungen (1), (3) einer allgemeinen Classe von algebraischen Gleichungen an, welche $\mathrm{Abel}$ in seiner Abhandlung ${ }^{1}$ ) "Sur une classe particulière d'équations résolubles algébriquement" hervorhebt. Wir behandeln zuerst die Gleichung (3). Wegen des Multiplicationstheorems ist $y_{a, a q}=\theta\left(y_{p, q}\right)$, wo $\theta$ eine rationale Function ist, es gehört somit die Gleichung $P\left(y^{2}\right)=0$ der Classe von irreductiblen Gleichungen an, deren Wurzeln sich nach dem folgenden Schema ordnen lassen:

(a)

$$
\begin{aligned}
& x_{1} \quad \theta x_{1} \theta^{2} x_{1} \ldots \theta^{n-1} x_{1} \\
& x_{2} \theta x_{2} \ldots \ldots \theta^{\mu-1} x_{2} \\
& x_{m} \quad 0 x_{m} \ldots \ldots \theta^{l c-1} x_{m} \text {. }
\end{aligned}
$$

Für solche setzt Abel nach Lagrange:

$$
\varphi^{(1)}(x)=\left(x-x_{1}\right) \ldots\left(x-\theta^{\mu-1} x_{1}\right)=x^{\mu \prime}+A_{1}^{\prime} x^{\mu-1}+\ldots+A_{\mu}^{\prime}=0,
$$

and zeigt, dass die Größen $A^{\prime}$ von einer Gleichung $m^{\text {ten }}$ Grades abhängen. Es sei in der That

$$
y_{1}=f\left(x_{1}, 0 x_{1}, \ldots, \theta^{\prime \prime-1} x_{1}\right)
$$

1) Oeurres Bd. I. 
eine symmetrische Function von $x_{1}, \theta x_{1}, \ldots, \theta^{u-1} x_{1}$, und es mögen $y_{1}, y_{2}, \ldots, y_{m}$ die Werte sein, die $y$ annimmt, wenn man $x_{1}$ durch $x_{2}, \ldots, x_{m}$ ersetzt. Dann sind die Coefficienten der Gleichung

$$
y^{m}+p_{1} y^{m_{-1}}+\ldots+p_{m}=0
$$

deren Wurzeln $y_{1}, y_{2} \ldots, y_{m}$ sind, rational.

Nach Galois' Theorie ist dies unmittelbar klar. Wir dürfen jedoch nicht vergessen, dass es zu A bel's Zeit ein ungeheuerer Schritt war, zu zeigen, dass für specielle Gleichungen gewisse Functionen der Wurzeln existieren, die, obschon nicht symmetrisch, doch rational sind. Hier, wie in anderer Beziehung, verstand $\mathrm{Abel}$ auf meisterhafte Weise die Fingerzeige des Altmeisters Lagrange zu verwerten; denn dieser hatte 1808 gezeigt $^{1}$, wie die Kreistheilungsgleichungen aufgelöst werdén können durch Benützung der Thatsache, dass die cyklischen Functionen der Wurzeln rational sind. Wir wollen deswegen etwas ausführlicher zeigen, wie $\mathrm{A}$ bel bewies, dass die Coefficienten $p_{1}, p_{2}, \ldots, p_{m}$ rationale Größsen sind.

Die Größen $\theta x_{1}, \theta^{2} x_{1}, \ldots$ sind rationale Functionen von $x_{1}$, somit ist $y_{1}$ eine rationale Function

von $x_{1}$.

$$
y_{1}=F\left(x_{1}\right)
$$

Indem wir $x_{1}$ durch $\theta x_{1}, \theta^{2} x_{1} \ldots$ ersetzen, erhalten wir

$$
y_{1}=F\left(x_{1}\right)=F\left(\theta x_{1}\right) \ldots=F\left(\theta^{n-1} x_{1}\right)
$$

und ähnlich

$$
y_{2}=F\left(x_{2}\right)=F\left(\theta x_{2}\right) \ldots=F\left(\theta^{n-1} x_{2}\right)
$$

somit

$$
\begin{aligned}
& y_{1}^{\nu}=\frac{1}{\mu}\left\{\left(F\left(x_{1}\right)\right)^{\nu}+\left(F\left(\theta x_{1}\right)\right)^{\nu}+\cdots+\left(F\left(\theta^{\mu-1} x_{1}\right)\right)^{\nu}\right\} \\
& y_{m}^{\nu}=\frac{1}{\mu}\left\{\left(F\left(x_{m}\right)\right)^{\nu}+\ldots \cdot \cdot+\left(F\left(\theta^{l-1} x_{m}\right)\right)^{\nu}\right\} \text {. }
\end{aligned}
$$

Die Summen $y_{1}^{v}+y_{2}^{v}+\ldots+\dot{y}_{m}^{*}=s_{\nu}$ sind rationale symmetrische Functionen aller Wurzeln $x_{2}$ und somit rational bekannt, folglich auch die Größen $p_{1}, p_{2}, \ldots, p_{m}$. 
Jeder der Coefficienten $A_{1}^{\prime}, A_{2}^{\prime}, \ldots, A_{\mu}^{\prime}$ ist somit Wurzel einer Gleichung $m^{\text {ten }}$ Grades mit rationalen Coefficienten.

$\mathrm{Abel}$ zeigt ferner, dass es genügt, eine einzige Wurzel einer einzigen Gleichung zu kennen, wobei er wieder genöthigt ist, einen langen Beweis zu geben, weil er die Thatsache nicht benuitzt, dass $y_{1}, A_{1}^{\prime}, A_{2}^{\prime}, \ldots$ dieselbe Gruppe haben. Sind die Gröluen $A_{1}^{\prime}, A_{2}^{\prime}, \ldots$ bekannt, so lässt sich die Gleichung (4) sehr leicht auflösen.

$\mathrm{Zu}$ dem Behufe setzt Abel mit Lagrange

$$
\psi\left(x_{1}\right)=\left(x_{1}+\alpha \theta x_{1}+\alpha^{2} \theta^{2} x_{1}+\ldots+\alpha^{n-1} \theta^{n-1} x_{1}\right)^{\prime \prime},
$$

wo $\alpha^{\mu}=1$ ist. Da nun $\psi\left(\theta^{u+k} x_{1}\right)=\psi\left(\theta^{k} x_{1}\right)$ ist, so sehen wir sofort, dass

$$
\psi\left(x_{1}\right)=\frac{1}{\mu}\left(\psi\left(x_{1}\right)+\psi\left(\theta x_{1}\right)+\ldots+\psi\left(\theta^{\mu-1} x_{1}\right)\right)
$$

also eine rationale symmetrische Function der Wurzeln von (4) und sonach bekannt ist.

Ersetzen wir in $A_{1}^{\prime}, A_{2}^{\prime}, \ldots, y_{1}$ durch $y_{2}, y_{3}, \ldots, y_{m}$, so geht $A^{\prime}$ über in $A^{(2)}, A^{(3)}, \ldots, A^{(m)}$ oder $\varphi^{(1)}$ in $\varphi^{(2)}, \varphi^{(3)}, \ldots, \varphi^{(m)}$. Daraus folgt, dass die Gleichung, deren Wurzeln durch die Tabelle $(a)$ dargestellt sind, dureh $m$ Gleichungen $\mu^{\text {ten }}$ Grades, deren Coefficienten von einer Gleichung $m^{\text {ten }}$ Grades abhängen, ersetzt werden kann. Es sollen nun diese Resultate auf die Gleichung (3) angewendet werden.

Hier ist $\mu=n-1, m=n+1$; somit hängt die Auflösung dieser Gleichung von einer Gleichung $(n+1)^{\text {ten }}$ Grades ab, deren Wurzeln die $(n+1)$ Werte einer symmetrischen Function $z$ für die verschiedenen Gruppen von Wurzeln

$$
y_{p, q} y_{2 p, 2 q} \cdots y_{(n-1) p,(n-1) q}
$$

sind:

Der Klarheit halber haben wir die Gleichung $P=0$ zuerst behandelt und gezeigt, wie deren Auflösung auf die Modulargleichungen $(n+1)^{\text {ten }}$ Grades führt. Wir wollen nun auf die Gleichung (1) zurückkommen, und zeigen, dass sie, wie schon oben behauptet wurde, algebraisch auflösbar ist, wenn wir die $y_{p, q}$ als bekannt ansehen. 
In der That sind in diesem Falle die Wurzeln

$$
x_{p, q}=\operatorname{sn}\left(\frac{v}{n}+\frac{4 p K+4 q K^{\prime}}{n}\right) \quad(p, q=0,1,2, \ldots, n-1)
$$

nach dem Additionstheorem rationale Functionen von $x_{0,0}$ und

$$
x_{p+p^{\prime}, q+q^{\prime}}=\theta_{p+p^{\prime}, q+q^{\prime}} x_{0,0}=\theta_{p, q} \theta_{p^{\prime}, q^{\prime}}\left(x_{0,0}\right)=\theta_{p^{\prime}, q^{\prime}} \theta_{p, q}\left(x_{0,0}\right),
$$

wo $\theta$ eine rationale Function bezeichnet.

Wir haben wiederum eine Tabelle wie vorher, nur ist in diesem Falle $m=\mu=n$. Wir bilden die Gleichung $n^{\text {ten }}$ Grades

$$
x^{n}+A_{1}^{\prime} x^{n-1}+\ldots+A_{n}^{\prime}=0
$$

und finden, dass die Coefficienten $A^{\prime}$ von der Gleichung

$$
y^{n}+p_{1} y^{n-1}+\ldots+p_{n}=0
$$

abhängen, wo $p_{1}, p_{2}, \ldots$ rational bekannt sind. Nun tritt aber ein Unterschied ein; die Wurzeln der Gleichung. (5) haben dieselben Eigenschaften, wie die der Gleichung (1), somit ist die Gleichung $F(x)=0$ algebraisch auflösbar.

Dass A bel durch das Studium Lagrange's vielfache Anregung erhielt, ist zweifellos, denn seinem scharfen Blicke ist es beispielsweise sicherlich nicht entgangen, dass La grange's Satz über die rationale Ausdrückbarkeit ähnlicher Functionen sich auf specielle Gleichungen ausdehnen lässt. Wir haben eben gesehen, dass er ihn für einen besonderen Fall beweist. Er hat weiters ein Kriterium der Auflösbarkeit, denn er behauptet, dass die Modulargleichungen $(n+1)^{\text {ton }}$ Grades nicht auflösbar sind. Was die Gleichungen vom PrimzahlGrade betrifft, ist Abel sicher im Rechte, denn in der schon erwähnten nachgelassenen Abhandlung sagt er, ,si une équation irréductible est resoluble algébriquement on pourra dans tous les cas trouver les racines à l'aide de la méthode de Lagrange, proposée pour la résolution des équations; savoir, en suivant la marche de Lagrange on doit parvenir à des équations qui aient au moins une racine qui puisse s'exprimer rationnellement par les coefficients." Es findet sich weiter z. B. in einer anderen Abhandlung.1) Lagrange's Satz, dass sich jede rationale Function der Wurzeln rational durch die n! wertige Function $u_{1} x_{1}+u_{2} x_{2}+\ldots+u_{n} x_{n}$ ausdrücken lässt, auf specielle Gleichungen ausgedehnt. Es mag hier nur hervorgehoben werden; dass Galois die Abel'schen Methoden sehr genau kennt, denn er hebt hervor, dass sich der letzterwähnte Satz bei A bel vorfindet; wie viel er ihm verdankt, mag ununtersucht bleiben.

1) Précis d'une theorie des fonctions elliptiques. 


\section{Jacobi.}

$\mathrm{Zu}$ der Modulargleichung gelangt Jacobi auf dem Wege der Transformation. Er stellt an die Spitze seines großen Werkes ${ }^{1}$ ): Problema, quod nobis proponimus, generale hoe est: "Quaeritur functio rationalis $y$ elementi $x$ ejusmodi, ut sit

$$
\frac{d y}{\sqrt{A^{\prime}+B^{\prime} y+C^{\prime} y^{2}+D^{\prime} y^{3}+E^{\prime} y^{4}}}=\frac{d x}{\sqrt{A+B x+C x^{2}+D x^{3}+E} \bar{x}^{4}} .
$$

Quod problema et multiplicationem videmus amplecti et transformationem. Das Problem reduciert sich auf die Bestimmung der Function

so dass

$$
y=\frac{a_{0}+a_{1} x+\ldots+a_{p} x^{p}}{b_{0}+b_{1} x+\ldots+b_{p} x^{p}}
$$

$$
\frac{d y}{\sqrt{1-y^{2} \cdot 1-\lambda^{2} y}}=\frac{d x}{M \sqrt{1-x^{2} \cdot 1-k^{2} x^{2}}}
$$

wo $k^{2}$ gegeben ist, und die Größen $a, b, \lambda$ und $M$ zu bestimmen sind. Die Ausführung dieses Problems hängt, wie Jacobi zeigt, von dem Werte von $\lambda$ ab. Er findet

$$
\xi=\frac{\lambda}{k^{n}}=\{\operatorname{snc} 4 \omega \operatorname{snc} 8 \omega \ldots \operatorname{snc} 2(n-1) \omega\}^{4},
$$

wo

$$
\omega=\frac{m K+m^{\prime} K^{\prime} i}{n}
$$

ist. Nun ist $\xi$ (sowie $\sqrt{\xi}$ ) eine symmetrische Function der Wurzeln

$$
y_{m, m^{\prime}} y_{2 m, 2 m^{\prime}} \cdots y_{(n-1) m,(n-1) m^{\prime}}
$$

der Gleichung $P\left(y^{2}\right)=0$. Diese Function genügt somit einer Gleichung $\left.{ }^{2}\right)(n+1)^{\text {ten }}$ Grades, $\mathfrak{u}$. zw. einer Modulargleichung.

Für $n=\mathbf{5}$ findet Jacobi die Gleichung:

$$
u^{6}-v^{6}+5 u^{2} v^{2}\left(u^{2}-v^{2}\right)+4 u v\left(1-u^{4} v^{4}\right)=0
$$

in welcher $v=\sqrt{\lambda}$ and $u=\sqrt{k}$.

Diese Gleichung wurde von Hermite als die Grundlage zur Auflösung der allgemeinen Gleichung fünften Grades benützt.

1) Fundamenta Nova, Königsberg 1829. Werke Bd. I.

2) $n$ ist als Primzahl vorausgesetzt. 
Ans demselben Grunde, wie bei der Function $\lambda$, ist

$$
\mu=(-1)^{\frac{n-1}{2}} \frac{(\operatorname{snc} c 4 \omega \operatorname{snc} 2(n-1) \omega)^{2}}{(\operatorname{sn}} \frac{\operatorname{4s} \ldots \operatorname{sn} 2(n-1) \omega)^{2}}{4 \omega}
$$

Wurzel einer Modulargleichung, oder, wie wir lieber sagen wollen, einer Transformationsgleichung.

Die Gleichung, welcher für $n=5 \approx=\frac{1}{M}$ genügt, nämlich

$$
(z-1)^{6}-4(z-1)^{5}+2^{8} k^{2} k^{2} z=0
$$

wurde von Brioschi benützt; sie ist eine Multiplicatorgleichung.

Noch eine dritte Gleichung, welche die Theorie der Transformation liefert, wollen wir hervorhen, nämlich

Wo

$$
\zeta^{6}-\frac{160}{k^{2} k^{\prime 2}} \zeta^{3}-2^{8} \frac{1-16 k^{2} k^{2}}{k^{4} k^{\prime 4}} \zeta+5 \cdot \frac{2^{8}}{k^{4} k^{\prime 4}}=0
$$

ist.

$$
\zeta=\left(\frac{c n 2 \omega}{c n 4 \omega}-\frac{c n 4 \omega}{c n 2 \omega}\right)^{2}=\frac{1}{M}\left(\sqrt{\frac{\lambda}{k}}+\sqrt{\frac{\lambda^{\prime}}{k^{\prime}}}\right)^{2}
$$

Sie dient Krońecker zur Auflösung der Gleichung fünften Grades durch elliptische Functionen. In Bezug auf die Gröhe $\frac{1}{M}$ macht Jacobi eine überaus wichtige Bemerkung. ${ }^{1}$ )

"Ces équations entre $k$ et $z$ jouissent d'une propriété remarquable. Savoir $\mathbf{n}$ étant un nombre premier quelconque, on peut exprimer linéairement la moitié des valeurs de $V_{\bar{z}}^{-}$au moyen de l'autre moitié. En effet si l'on désigne par $z, z^{\prime}, z^{\prime \prime}, \ldots z^{(n)}$ les racines de l'équation du $(n+1)^{\text {ème }}$ degré trouvée entre $z$ et $k$, on aura:

$$
\begin{aligned}
& \sqrt{z}=\sqrt{(-1)^{\frac{n-1}{2}} n A} \\
& \sqrt{z^{\prime}}=A+A_{1}+A_{2}+\ldots+A_{\frac{n-1}{2}} \\
& \sqrt{z^{\prime \prime}}=A+\alpha A_{1}+\alpha^{4} A_{2}+\ldots+\alpha^{\left(\frac{n-1}{2}\right)^{2}} A_{\frac{n-1}{2}} \\
& \sqrt{z^{\prime \prime \prime}}=A+\beta A_{1}+\beta^{4} A_{2}+\ldots+\beta^{\left(\frac{n-1}{2}\right)^{2}} \frac{A_{n-1}}{2}
\end{aligned}
$$

1) Suite des Notices sur les fonctions elliptiques. Oeurres Bd. I. 
$x, \beta$ etc. étant les racines imaginaires de l'équation $x^{n}-1=0$. Donc on peut exprimer linćairement les racines carrées des $n+1$ racines par d'autres quantités dont le nombre n'est que $\frac{n+1}{2}$. Cela donne le théorème enoncé, un des plus importants dans la theorie algébrique de la transformation et de la division des fonctions elliptiques." Evr bemerkt weiter, dass man dasselbe Theorem für die Größen $z \lambda, z \lambda^{\prime} \ldots$ hat. Diese prophetischen Worte, welche cin schönes Beispiel von Jacobi's genialer Einsicht sind, wurden durch Brioschi und Kronecker verwirklicht.

Ehe wir J a cobi verlassen, wollen wir noch eine Resolvente ${ }^{1}$ ) erwähnen, die er entdeckte.

Ist

$$
\begin{aligned}
& t_{1}=x_{0} x_{1}+x_{1} x_{2}+x_{2} x_{3}+x_{3} x_{4}+x_{4} x_{0}=\left(\begin{array}{lll}
0 & 1234
\end{array}\right) \\
& t_{2}=\left(\begin{array}{lll}
02413
\end{array}\right)
\end{aligned}
$$

so ist nicht nur

$$
y^{2}=\left(t_{1}-t_{2}\right)^{2}
$$

sondern aach $y$ Wurzel einer Gleichung sechsten Grades. Die Coefficienten der Gleichung in

$$
y=t_{1}-t_{2}
$$

sind rational in den Coefficienten der gegebenen Gleichung fünften Grades und der Quadratwurzel ihrer Discriminante $\Delta$. In der That verändert sich nur das Vorzeichen von $y$ fürr die Substitutionen der metacyclischen Gruppe

$$
|v, a v+b| \quad(\bmod .5) .
$$

Die Jacobi'sche resolvierende Function, welche somit der halb metacyclischen Gruppe angehört, unterscheidet sich von den Lagrange'schen und Malfatti'schen, welche metacyclische Functionen sind.

Jacobi fand ferner, dass $y$ einer Gleichung von der Form

$$
y^{6}+\alpha y^{4}+\beta y^{2}+\gamma=32 \sqrt{\Delta}
$$

genügt.

Die Resolventen von $\mathrm{Malfatti}$ und $\mathrm{J}$ a co bi hängen mit einander durch die einfache Gleichung

$$
y^{2}=-20\left(z-\frac{5}{4} a^{2}--\frac{5}{3} c\right)
$$

zusammen. 
Cayley ${ }^{1}$ ) berechnete, ohne von den Resultaten Malfatti's und J a c o b i's Kenntnis zu haben, die Coefficienten der Gleichung (a). Wir wollen hier noch hervorheben, dass auch Kronecker, wie Jacobi, eine halbmetacyclische Function verwendete, um seine Resolvente $\mathrm{zu}$ bilden.

\section{Galois.}

Die immer wiederkehrende Frage bei der Auflösung algebraischer Gleichungen, welche Größen unter gegebenen Umständen rational sind, hatte Lagrange für die allgemeine Gleichung beantwortet; Galois dehnte die Resultate und Methoden von Lagrange auf specielle Gleichungen aus.

Als Basis seiner Untersuchungen, nach dem Vorgange Lagrange's und Abel's, legte er die $n$ ! wertige Function

$$
V_{0}=u_{0} x_{0}+u_{1} x_{1}+\ldots+u_{n-1} x_{n-1}
$$

der Wurzeln $x_{0}, x_{1}, \ldots, x_{n-1}$ einer irreductiblen Gleichung $n^{\text {ten }}$ Grades $f(x)=0 \mathrm{zu}$ Grunde, in welcher $u_{0}, u_{1}, \ldots, u_{n-1}$ unbestimmte Größen sind. Ist die Gleichung von $n$ ! Grade

$$
\left(V-V_{0}\right)\left(V-V_{1}\right) \ldots=0
$$

reductibel, wenn wir gewisse Größen $\mathfrak{R}^{\prime}, \mathfrak{R}^{\prime \prime} \ldots$ als bekannt adjungieren, so möge

$$
V=\left(V-V_{a}\right)\left(V-V_{\imath}\right)\left(V-V_{e}\right) \ldots=0
$$

ein irreductibler Factor sein, wo $V_{a}, V_{b}, V_{c}, \ldots$ die versehiedenen Werte von $V_{0}$ nach Anwendung der Substitutionen $G$ sind. Diese Substitutionen spielen nun für die vorgelegte Gleichung $f(x)=0$ genau dieselbe Rolle, wie die symmetrische Gruppe für die allgemeine Gleichung, nämlich :

$1^{0}$ Jede rational (bekannte) Function der Wurzeln bleibt nach Anwendung der Substitutionen der Gruppe $G$ (Gruppe der Gleichung) unverändert;

$2^{\circ}$ Jede rationale Function der Wurzeln, welche für die Substitutionen der Gruppe $G$ unverändert bleibt, ist rational bekannt.

Diese Eigenschaften definieren die Gruppe $G$ für den Rationalitätsbereich $\mathfrak{R}^{\prime}, \mathfrak{R}^{\prime \prime} \ldots$

Die von Galois herrührende Einführung des Begriffes der (G a lo is'schen) Gruppe einer Gleichung gehört zu. den wichtigsten Fortschritten auf dem Gebiete der Algebra in diesem Jahrhundert,

$\left.{ }^{1}\right)$ On a new auxiliary equation in theory of equations of the $5^{\text {th }}$ degree Phil. Trans, vol 152. 1861. 
Adjungiert man nun die Wurzeln der rationalen Resolvente

$$
\left(\varphi-\varphi_{1}\right)\left(\varphi-\varphi_{2}\right) \ldots=0
$$

wo $\varphi_{1}, \varphi_{2}, \ldots$ die verschiedenen Werte, die $\varphi_{1}$ für $G$ annimmt, sind, so reduciert sich $G$ auf die größte invariante Untergruppe $H$, welche $\varphi_{1}, \varphi_{2}, \ldots$ unverändert lässt. Gehört $\varphi_{1}$ zu der Gruppe $H$, so sind $\varphi_{1}, \varphi_{2}, \ldots$ rationale Functionen von einander. Umgekehrt, hat $G$ eine invariante Untergruppe $I$ vom Index $p$, so existiert stets eine Gleichung $p^{\text {ten }}$ Grades

$$
\varphi=\left(\varphi-\varphi_{1}\right) \ldots\left(\varphi-\varphi_{1}\right)=0,
$$

deren Wurzeln rationale Functionen von einander sind. Ist eine darunter $\varphi_{1}$ adjungiert, so reduciert sich $G$ auf $H$. Ist $p$ eine Primzahl, so ist, wie A bel gezeigt hat, $\varphi=0$ auflösbar. Daraus folgt durch Wiederholung folgender Satz:

Die Gleichung $f(x)=0$ ist algebraisch auflösbar, wenn ihre Gruppe $G$ eine invariante Untergruppe vom Primzahl-Index besitzt, welche wiederum eine solche Untergruppe besitzt u.s.f.

Dass diese Bedingung nicht nur hinreichend, sondern auch nothwendig für die algebraische Auflösbarkeit ist, lässt sich leicht unter Benützung eines Satzes von Camille Jorda $n$ beweisen. Der erwähnte Satz lautet: Wird die Gruppe $G$ einer Gleichung $I^{\prime}(x)=0$ durch die Adjunction der Wurzeln $z_{1}, z_{2}, \ldots$ einer Gleichung $H(x)=0$, deren Gruppe $G_{1}$ sein mag, auf den $p^{\text {ten }}$ Theil ihrer Substitutionen reduciert, so wird auch $G_{1}$ durch die Adjunction der Wurzeln $x_{1}, x_{2}, \ldots$ der Gleichung $F(x)=0$ auf den $p^{\text {ten }}$ Theil jihrer Substitutionen reduciert.

In der 'That, ist die Gleichung, deren Gruppe $G$ sein mag; algebraisch auflösbar, so muss man imstande sein, die Gruppe $G$ nach und nach zu reducieren, so dass sie schließlich nur aus der dentischen Substitution besteht, und zwar dadurch, dass man den Rationalitätsbereich durch successive Adjunction der Wurzeln binomischer Gleichungen von dem Typus

$$
y^{p}-A=0
$$

erweitert, wo $p$ eine Primzahl und $A$ eine in dem augenblicklichen Rationalitätsbereich gelegene Function der Wurzeln ist. Wir nehmen an, dass die $p^{\text {ten }}$ Einheitswurzeln schon adjungiert sind. Die Gleichung (1) ist eine Abel'sche; ihre Gruppe besteht aus den cyklischen Substitutionen der $p$ Wurzeln $y_{1}, y_{2}, \ldots, y_{p}$; sie ist somit einfach. Reduciert sich nun $G$ durch die Adjunction von $y_{1}, y_{2}, \ldots$ auf $G^{\prime}$, so muss sich $G$ durch Adjunction von $x_{1}, x_{2}, \ldots, x_{n}$ auf die identische Substitution reducieren, da $p$ eine Primzahl ist. Somit sind $y_{1}, y_{2}, \ldots$ rationale Functionen von $x_{1}, x_{2}, \ldots$, und $G^{\prime}$ ist eine invariante untergruppe rom Index $p$. Die oben erwähnte Bedingung der Auf- 
lösbarkeit ist somit nothwendig. Für einen Primzahlgrad besteht die Gruppe einer algebraisch auflösbaren Gleichung aus den Substitutionen

$$
[\nu, a \nu+b] .
$$

Wir sehen daraus, dass die Methode von Lagrange in diesem Falle stets erfolgreich sein muss.

Galois wendet seine Methode auf die Periodentheilungsgleichung ${ }^{1)} P\left(y^{2}\right)=0$ für die ungerade Primzahl $n$ an.

In dem berühmten Briefe, welchen er am Vorabend seines unglïckseligen Duells an seinen Freund Auguste Chevalier richtete, erklärte Galois, ohne Beweis, dass die Gruppe dieser Gleichung aus den Substitutionen

$$
|p, q ; a p+b q, c p+d q| a d-c b \neq 0(\bmod n)
$$

besteht.

Die Gruppe der Modulargleichung würde alsdann aus den Substitutionen

$$
z, \frac{a z+b}{c z+d}
$$

bestehen. Die Gruppe $B$ ) enthält eine invariante Untergruppe $B^{\prime}$ vom Index 2, welche aus denjenigen Substitutionen besteht, wofür

$$
a d-b c \equiv 1(\bmod n)
$$

ist.

Galois behauptet nun, dàss die Gruppe $B^{\prime}$, abgesehen von dem Falle $n=3$, keine invariante Untergruppe besitzt, wohl aber Untergruppen vom Index $n$, allerdings nur für die Fälle $n=5,7,11$.

Diese Behauptungen blieben lange Zeit unbewiesen.

Erst 1853 nahm Betti ${ }^{2}$ ) dieselben auf. Er zeigte, dass die Gruppe der Theilungsgleichung $P=0$ mindestens in $A$ enthalten ist, bewies die Galois'schen Behauptungen bezüglich der Gruppe $B^{\prime}$, und gab die Gruppen rom Index 5,7 und 11 thatsächlich an. Es blieb noch übrig zu beweisen, dass die Gruppe der Theilungsgleichung nicht nur in $A$ enthalten, sondern dass sie mit $A$ identis ch sei. Dies wurde erst im Jahre 1871 von Sylow ${ }^{3}$ ) gezeigt.

Insofern diese Resultate die Theorie der Gleichung 5. Grades berühren, will ich den Beweis andeuten.

1) S. unter A be l, Gleichung (3).

2) Annali di Scienze Matematiche e Fisiche. Bd. 4.

3) Christiania Videnskabsselskabs Forhandlinger 1871.

Weber; Acta Mathematica, Bd, VI., sowie Elliptische Functionen, Braunschweig 1891. 
Bezeichnen wir, wie zuvor, die Gruppe der linearen Substitutionen mit $A$ und deren Untergruppe, die aus den Substitutionen besteht, für welche

$$
a d-b c \equiv 1(\bmod n)
$$

ist, mit $A^{\prime}$; endlich die Gruppe der Theilungsgleichung $P=0 \mathrm{mit}$ $A_{0}$, so soll gezeigt werden, dass $A_{0}=A$ ist.

Aus dem Additions- und Multiplicationsthoreme folgt unmittelbar, dass $A_{0}$ in $A$ enthalten ist.

Betrachten wir nun die Wurzel $y_{p, q}$ und $k^{2}$ als Functionen von $\omega=\frac{K^{\prime} i}{K}$. Die Substitution

$$
\left|\omega, \frac{\gamma+\delta \omega}{\alpha+\beta \omega}\right|
$$

verwandelt $x_{p, q}$ in $x_{\alpha p+\gamma q, \beta p+\delta q}$, während $k^{2}$ unverändert bleibt, wenn wir

$$
\alpha \equiv \delta \equiv 1 ; \beta \equiv \gamma \equiv 0(\bmod 8) \text { und } \alpha \delta-\beta \gamma=1
$$

nehmen.

Daraus folgt, dass jede rationale Gleichung zwischen den Größen $y_{p, q}$ und $k^{2}$, die Substitutionen $A^{\prime}$ gestattet. Die Gruppe $A^{\prime}$ ist somit in der Gruppe von $P=0$ enthalten, auch sogar wenn diese nach Adjunction beliebiger Constanten reduciert ist. Wir adjungieren jetzt $s=e^{\frac{2 \pi i}{n}}$, die Gleichung

$$
\Phi=\sum_{p=0}^{p=n-1} \varepsilon^{4 p q} x_{p, q}=0
$$

ist nunmehr eine rationale Gleichung. Da die Substitution $|p, q ; p, m q|$ in $\Phi=0$ nicht gestattet ist, wenn nicht $m \equiv 1,(\bmod n)$ ist, so ist $A^{\prime}$ die Gruppe von $P=0$ nach Adjunction $\operatorname{der}^{\text {ten }}$ Einheitswurzeln. Es ist nun leicht zu zeigen, dass $A_{0}=A$ ist. Es sei

$$
f(x)=\frac{x^{n}-1}{x-1}=0 .
$$

Die zwei Gleichungen $\Phi=0$ und $f=0$ haben nur die Wurzel $\varepsilon$ gemeinsam, somit ist $\varepsilon$ in den $y_{p, q}$ rational. Die Adjunction der Wurzeln von $P=0$ reduciert somit die Gruppe von $f=0$ auf die identische Substitution; umgekehrt reduciert die Adjunction von $\approx$ nach Jordan's Satz die Gruppe $A_{0}$ auf ein $(n-1)^{\text {tel }}$. Somit ist $A_{0}=A$. 
Betrachten wir jetzt die Gruppe der Modulargleichung, welche nach dem vorhergehenden aus den Substitutionen

B)

$$
\left|z, \frac{a z+\frac{1}{c z} \mid d}{+d}\right| a d-b c \neq 0(\bmod n)
$$

oder nach Adjunction von $\varepsilon$ aus $B^{\prime}$ besteht.

Es muss jedoch die Adjunction einer zweiwertigen Function genügen, um $B$ auf $B^{\prime}$ zu reducieren. Eine solche Function ist z. $B$.

$$
s=\sum_{r} \mathbf{\Sigma}^{r},
$$

wo $r$ die quadratischen Reste von $n$ durchläuft.

In der That geht $\varepsilon$ nach der Substitution

$$
|p, q ; a p+c q, b p+d q| \quad a d-b c \equiv m(\bmod n)
$$

in $\varepsilon^{m}$ über.

Somit genügt die Adjunction von $\sqrt{(-1)^{\frac{n-1}{2}}} n$.

Für $n=5$ ist $s=\varepsilon^{1}+\varepsilon^{4}=-\frac{-1+\sqrt{5}}{2}$.

Die Gruppe $B$ enthält für $n=5120$ Substitutionen; sie ist mit den Resolventen von Lagrange und Malfatti holoedrisch isomorph. Daraus folgt die Wichtigkeit der Modulargleichung für die Gleichung 5. Grades. Aus dieser Thatsache folgt, dass $B^{\prime}$ für diesen Fall keine invariante Untergruppe, wohl aber eine Untergruppe $C$ vom. Index 5 besitzt. Sie besteht, wie Betti zeigte, aus den 12 Substitutionen, welche dureh

$$
|z, 4 z| \quad\left|z, \frac{1}{z}\right| \quad\left|z, 3 \frac{z+1}{z-1}\right|
$$

erzeugt werden.

Die Indices

$$
\infty 01234
$$

verwandeln sich nach Anwendung dieser Substitutionen in

$$
\begin{array}{cccccc}
\infty & 0 & 4 & 3 & 2 & 1 \\
0 & \infty & 1 & 3 & 2 & 4 \\
3 & 2 & \infty & 4 & 1 & 0 .
\end{array}
$$

Wir bemerken noch, dass die Paare

$$
\infty \begin{array}{lll}
\infty & 0 & 14 \\
23
\end{array}
$$

durch die Substitutionen $C$ auseinandergerissen werden. 
Eine Function der Wurzeln

$$
\begin{array}{lllllll}
v_{\infty} & v_{0} & v_{1} & v_{2} & v_{3} & v_{4}
\end{array}
$$

emer Transformationsgleichung, wic z. B.

$$
\left(v_{\infty}-v_{0}\right)\left(v_{1}-v_{4}\right)\left(v_{2}-v_{3}\right),
$$

bleibt somit für $C$ unverändert, und ist darnach Wurzel einer Gleichung vom fünften Grade, deren Coefficienten rational in $k^{2}$ und $\sqrt{5}$ sind.

\section{Hermite.}

Nach den eben erwähnten gruppentheoretischen Ergebnissen offnen sich zwei Wege, um die allgemeine Gleichung fünften Grades aufzulösen; entweder kann man der allgemeinen Gleichung tünften Grades durch Transformation eine solche Form zu geben suchen, dass sich dieselbe mit der Resolvente fünften Grades einer Transformationsgleichung identificieren lässt, oder eine Resolvente sechsten Grades der allgemeinen Gleichung fünften Grades suchen, welche mit einer Transformationsgleichung identificiert werden kann. Den ersten Weg wählten Hermite und Brioschi, den zweiten Kronecker.

Da Hermite ${ }^{1}$ ) der erste war, der hierüber etwas veröffentlichte, so wenden wir uns zunächst seiner Methode zu; unsere Betrachtungen lassen sich um so kürzer fassen, als schon alle nothwendigen Hilfsmittel entwickelt wurden.

Nach den Beweisen von $\mathrm{R}$ uffini und $\mathrm{Abel}$ ist die Gleichung: fünften Grades mit Hilfe von reinen Gleichungen nicht auflösbar; es entsteht nun die Frage, wie eine Auflösung dieser algebraisch unauflösbaren Gleichungen beschaffen sein soll. Hermite suchte diese folgenschwere Frage durch folgende Überlegungen zu beantworten: "Cette impossibilité manifeste en effet la necessité d'entroduire quelque élément analytique nouveau dans la recherche de la solution, et à ce titre il semble naturel de prendre comme auxiliare les racines de l'équation dont nous venons de parler (nämlich Jerrard's Form, $x^{5}-x-a=0$ ). 'Toutefois, pour legitimer véritablement son emploi comme élément essentiel de lar ésolution de l'équation générale, il restait à voir si cette simplicité de forme permettait effectivement d'arriver à quelque notion sur la nature de ces racines, de manière à saisir ce qu'il y a de propre et d'essentiel dans le mode d'existence de ces quantités, dont on ne sait jusqu'ici rien autre chose, si ce n'est qu'elles ne s'expriment point par radicaux. Or il est bien remarquable que l'équation de M. Jerrard se prête avec la plus grande facilité à cette recherche,

1) Sur la résolution de l'équation du cinquième degré. Comptes Rendus. 13d. 46. (März 1858.) 
et soit même, dans le sens que nous allons expliquer, susceptible d'une veritable résolution analytique. On peut en effet concevoir la question de la résolution des equations algébrique sous un point de vue différent de celui qui depuis longtemps a été indiqué par la résolution des equations des quatre premiers degrés, et au quel on s'est surtout attaché. Au lieu de chercher à représenter par une formule radicale à determinations multiples le system des racines étroitement liées entre elles lorsqu'on les considère comme fonctions des coefficients, on peut, ainsi que l'exemple eu a été donné dans le troisième degré, chercher, en introduisant des variables auxiliares, à obtenir les racines séparément exprimées par autant de fonctions destinctes et uniformes relatives à ces nouvelles variables. Dans le cas dont nous venons de parler où il s'agit de l'équation

$$
x^{3}-3 x+2 a=0,
$$

il suffit, comme on sait, de représenter le coefficient $a$ par le sinus d'un are $\alpha$ pour que les racines se séparent en ces trois fonctions bien determinées

$$
2 \sin \frac{\alpha}{3} \quad 2 \sin \frac{\alpha+2 \pi}{3} \quad 2 \sin \frac{\alpha+4 \pi}{3} .
$$

Or c'est un fait tout semblable que nous avons à exposer relativement à l'equation

$$
x^{5}-x-a=0 .
$$

Seulement, au lieu des sinus, ou cosinus, ce sont les transcendentes elliptiques qu'il sera nécessaire d'introduire."

In der That findet $\mathrm{Hermite}$, dass die Modulargleichung

$$
u^{6}-v^{6}+5 u^{2} v^{2}\left(u^{2}-v^{2}\right)+4 u v\left(1-u^{4} v^{4}\right)=0
$$

die Resolvente

$$
\Phi^{5}-2^{4} .5^{3} u^{4}\left(1-u^{8}\right)^{2} \Phi-2^{6} \sqrt{5^{5}} u^{3}\left(1-u^{8}\right)^{2}\left(1+u^{8}\right)=0
$$

besitzt, wo $\Phi$ die schon erwähnte resolvierende Function

$$
\Phi(\omega)=\left(v_{\infty}-v_{0}\right)\left(v_{1}-v_{4}\right)\left(v_{2}-v_{3}\right)
$$

ist. Hermite führt die zwei Functionen

und

$$
\varphi(\omega)=u=\sqrt[4]{k}=\sqrt{2} q^{\frac{1}{8}} \frac{\Sigma q^{2 m^{2}+m}}{\Sigma q^{m^{3}}} \quad(m=-\infty \ldots+\infty)
$$

$$
\psi(\omega)=\sqrt[4]{k^{\prime}}
$$


ein, so dass also

$v_{\infty}=\varphi\left(\frac{\omega}{5}\right), \quad v_{0}=-\varphi(5 \omega) \quad v_{\frac{1}{2}}=\varphi\left(\frac{\omega+16 \lambda}{5}\right) \quad(\lambda=1,2,3,4)$ ist.

Die Gleichung (3) nimmt die Jerrard'sche Form an, wenn

$$
\begin{aligned}
\Phi & =\sqrt[4]{2^{4} \cdot 5^{3}} k^{1 / 4} k^{\prime} \cdot x \\
a & =\frac{2}{\sqrt[4]{5^{5}}} \frac{1+k^{2}}{k^{\prime} \sqrt{k}}
\end{aligned}
$$

gesetzt wird. Setzt man ferner

$$
A=\frac{\sqrt[4]{5^{5}}}{2} a
$$

so ist $k$ durch die Gleichung

$$
k^{4}+A^{2} k^{3}+2 k^{2}-A^{2} k+1=0
$$

bestimmt, deren Wurzeln die Functionen

$$
\operatorname{tang} \frac{\alpha}{4}, \quad \operatorname{tang} \frac{\alpha+2 \pi}{4}, \quad \operatorname{tang} \frac{\pi-\alpha}{4}, \quad \operatorname{tang} \frac{3 \pi-\alpha}{4}
$$

sind, wenn

ist.

$$
\sin \alpha=\frac{1}{4 A^{2}}
$$

Somit hat Hermite sein Ziel erreicht, denn es ist

$$
x_{\nu}=\frac{1}{\sqrt{2^{4} \cdot b^{3}}} \frac{\Phi(\omega+16 \nu)}{\varphi(\omega) \psi^{4}(\omega)}, \quad \nu=0,1,2,: s, 4 .
$$

"C'est done la résolution de l'équation, en tant que les racines se trouvent représentées separément par des fonctions uniformes".

\section{Brioschi.}

In demselben Jahre, in welchem Hermite die eben besprochene Abhandlung veröffentlichte, lief $\mathrm{Br}$ i os $\mathrm{chi}^{1}$ ) eine zweite Auflösung der allgemeinen Gleichung fünften Grades erscheinen.

1) Annali di Matematica. Bd. I. (Mai 1858).

Atti dell' I. R. Istituto Lombardo. Bd. I. 1858. 
Er nimmt, wie wir schon erwähnt haben, anstatt der Modulargleichung die Multiplicatorgleichung, nämlich

$$
(z-1)^{6}-4(z-1)^{5}+2^{8} k^{2} k^{2} z=0 .
$$

Vermittelst derselbeu resolvierenden Funetion, die Hermite gebrauchte,

$$
x_{r}=\left(z_{\infty}-z_{r}\right)\left(z_{r+2}-z_{r+3}\right)\left(z_{r+4}-z_{r+1}\right) \quad r=0,1,2,3,4
$$

erhält Brioschi die Resolvente

$$
x\left(x^{2}+5^{2} \cdot 2^{8} k^{2} k^{\prime 2}\right)^{2}=5^{2} \sqrt{5} \cdot 2^{22} k^{4} k^{\prime 4}\left(1-4 k^{2} k^{\prime 2}\right) .
$$

Setzt man nun

$$
\frac{2}{5} y=1-\frac{x^{2}}{5^{2} \cdot 2^{8} k^{2} k^{2}}
$$

so ergibt sich

$$
y^{5}-\frac{5}{2} y^{4}-\frac{\left(1-k^{2} k^{2}\right)^{2}}{2 k^{2}}=0
$$

durch deren Vergleichung mit Jerrard's Form

$$
y^{5}-\frac{5}{2} y-a=0
$$

sich für $k$ die Gleichung

$$
\left(1-4 k^{2} k^{\prime 2}\right)^{2}=2 a k^{2} k^{\prime 2}
$$

ergibt.

Nun ist aber die Multiplicatorgleichung nur ein Individuum einer Classe von Transformationsgleichungen, deren merkwürdige Eigenschaft von J a cobi hervorgehoben wurde. ${ }^{1}$ )

Die Wurzeln einer Gleichung $F(x)=0$ dieser Classe mügen für den Fall $n=5$ mit

$$
x_{\infty}, x_{0}, x_{1}, x_{2}, x_{3}, x_{4}
$$

bezeichnet werden; alsdann bestehen nach Jacobi die Relationen:

$$
\begin{gathered}
\sqrt{x_{\infty}}=a_{0} \sqrt{5}, \quad \sqrt{x_{m}}=a_{0}+\varepsilon^{m} a_{1}+\varepsilon^{4 m} a_{2}, \\
\left(\varepsilon=e^{\frac{2 \pi i}{5}}, \quad m=1,2,3,4\right),
\end{gathered}
$$

1) Siehe Seite 53 . 
aus denen durch Elimination folgt:

$$
\begin{aligned}
& \sqrt{x_{0}}+\sqrt{x_{1}}+\sqrt{x_{2}}+\sqrt{x_{3}}+\sqrt{x_{4}}=\sqrt{5 x_{\infty}} \\
& \sqrt{x_{0}}+\varepsilon^{2} \sqrt{x_{1}}+\varepsilon^{4} \sqrt{x_{2}}+\varepsilon \sqrt{x_{3}}+\varepsilon^{3} \sqrt{x_{4}}=0 \\
& \sqrt{x_{0}}+\varepsilon^{3} \sqrt{x_{1}}+\varepsilon \sqrt{x_{2}}+\varepsilon^{4} \sqrt{x_{3}}+\varepsilon^{2} \sqrt{\overline{x_{4}}}=0
\end{aligned}
$$

Stellt man nun die durch die Gleichungen (1) bestimmte Gleichung $F(x)=0$, sowie deren Resolvente fünften Grades auf, so kann man durch bloßen Vergleich die Resolvente fünften Grades einer bestimmten Gleichung dieser Classe sofort niederschreiben.

Brioschi fand, dass die Gleichung $F(x)=0$ die bemerkenswert einfache Form

$$
\begin{gathered}
F(x)=(x-a)^{6}-4 a(x-a)^{5}+10 b(x-a)^{3}-c(x-a)+ \\
5 b^{2}-a c=0
\end{gathered}
$$

hat, wo

$$
\begin{aligned}
& a=a_{0}^{2}+a_{1} a_{2} \\
& b=8 a_{0}^{4} a_{1} a_{2}-2 a_{0}^{2} a_{1}^{2} a_{2}^{2}+a_{1}^{3} a_{2}^{3}-a_{0}\left(a_{1}^{5}+a_{2}^{5}\right) . \\
& \begin{aligned}
& c= 320 a_{0}^{6} a_{1}^{2} a_{2}^{2}-160 a_{0}^{4} a_{1}^{3} a_{2}^{3}+20 a_{0}^{2} a_{1}^{4} a_{2}^{4}+6 a_{1}^{5} a_{2}^{5}- \\
& \quad \quad-4 a_{0}\left(a_{1}^{5}+a_{2}^{5}\right)\left(320 a_{0}^{4}-20 a_{0}^{2} a_{1} a_{2}+5 a_{1}^{2} a_{2}^{2}\right)+a_{1}^{10}+a_{2}^{1}
\end{aligned}
\end{aligned}
$$

ist.

Benützt man die schon oft erwähnte resolvierende Function

(4) $y_{r}=\left(x_{\infty}-x_{r}\right)\left(x_{r+2}-x_{r+3}\right)\left(x_{r+4}-x_{r+1}\right) \quad r=0,1,2,3,4$,

so erhält man

$$
y^{5}+p_{1} y^{4}+p_{2} y^{3}+p_{3} y^{2}+p_{4} y+p_{5}=0
$$

wo

$$
\begin{aligned}
& p_{1}=-5 \cdot \sqrt{5} \cdot 4 b \\
& p_{2}=-5^{2} \cdot 2\left(a c-3 b^{2}\right) \\
& p_{3}=-5^{2} \sqrt{5} \cdot 4 b\left(a c-5 b^{2}\right) \\
& p_{4}=-5^{3}\left\{15 b^{4}-a^{2} c^{2}+2 a b^{2} c-4\left(a c-3 b^{2}\right)^{2}\right\} \\
& p_{5}=-\sqrt{\Delta}
\end{aligned}
$$

wo $\Delta$ die Discriminante von $F(x)$ ist.

Für die Multiplicatorgleichung z. B. ist

$$
a=0, \quad b=0, \quad c=-2^{8} k^{2} k^{2} .
$$


Brioschi entdeckte später noch in dem gleichen Jahre, veranlasst durch eine Mittheilung von Hermite, dass die Gleichung (3) eine noch einfachere Resolvente hat, welche man erhält, indem man anstatt $y_{r}$

$$
\sqrt{y_{r}}=\left\{\left(x_{\infty}-x_{r}\right)\left(x_{r+2}-x_{r+3}\right)\left(x_{r+4}-x_{r+1}\right)\right\}^{1 / 2}
$$

verwendet.

Hermite bemerkte nämlich, dass Brioschi's Resolvente sich vermittelst der Transformation

in

$$
\sqrt{x}=4 y \sqrt[4]{5}
$$

$$
y^{5}+5 k^{2} k^{\prime 2} y-2 k^{2} k^{\prime 2}\left(1-2 k^{2}\right)=0
$$

verwandeln lässt. Darauf vermuthete Brioschi, dass nicht nur $x_{\infty}, x_{0}, \ldots$, sondern auch $V \overline{x_{\infty}}, \sqrt{x_{0}}, \ldots$ eindeutige Functionen von $q$ sind. Und in der That findet man, wenn man

$$
z_{r}=\frac{1}{\sqrt{5}}\left\{\left(x_{\infty}-x_{r}\right)\left(x_{r+2}-x_{r+3}\right)\left(x_{r+4}-x_{r+1}\right)\right\}^{1 / 2} \quad(r=0,1,2,3,4)
$$

setzt, dass die Resolvente die äuberst einfache Gestalt

$$
y^{5}+10 b y^{3}+5\left(q b^{2}-4 a c\right) y-\sqrt[4]{5}=0
$$

annimmt. Die Existenz dieser Resolvente ergibt sich a priori nach der Theorie von $\mathrm{Kronecker,} \mathrm{über} \mathrm{dessen} \mathrm{in} \mathrm{diese} \mathrm{Epoche}$ fallenden Arbeiten einige Bemerkungen folgen sollen.

\section{Kronecker.}

Ganz unabhängig von Hermite und Brioschi, hatte sich Kronecker ${ }^{1}$ ) damals schon seit einiger Zeit mit der Auflösung der allgemeinen Gleichung fünften Grades beschäftigt. Seine Untersuchungen hatten ihn auch auf eine Methode geführt, welche sich auf die Jerrard'sche Form stïtzte. Da er jedoch eine Auflösungsmethode suchte, welche auf Gleichungen von höheren Graden angewendet werden könnte, wurde er veranlasst, jede Methode zu verwerfen, welche eine vorhergehende Reduction der Coefficienten fordern würde. Er wollte ferner die Auflösung nur so weit geführt sehen, als dies mit Hilfe von natürlichen Irrationalitäten möglich wäre; d. h. Irrationalitäten, welche sich rational durch Wurzeln der gegebenen Gleichung darstellen lassen. Es sollte somit der

1) Comptes Rendus 1858.

Monatsberichte der Berliner Akad. 1861.

Monatsh. f. Mathematik u. Physik, VI, Jahrg. 
A bel'sche Satz für algebraisch auflösbare Gleichungen auch für die Auflösung algebraisch nicht auflösbarer Gleichungen maßgebend sein. Dieses Postulat, welches ganz im Einklang mit den algebraischen Anschauungen Kronecker's steht, zwang ihn, Resolventen, welche nur von einem Parameter abhängen, aus dem Kreis seiner Untersuchungen auszuschließen. Die Gleichung fünften Grades besitzt nämlich keine rationale Resolvente, deren Coefficienten von weniger als zwei Parametern abhängen. Unter diesem Gesichtspunkte gewinnt das Jacobi'sche Theorem bezüglich der Quadrat. wurzeln der Multiplicatorgleichungen seine grolje Bedeutung, denn es lassen sich rationale Functionen der Wurzeln der allgemeinen Gleichung fünften Grades finden, welche die Jacobi'schen Relationen

$$
\sqrt{x_{\infty}}=a_{0} \sqrt{5} ; \quad \sqrt{x_{m}}=a_{0}+a^{m} a_{1}+\alpha^{4 m} a_{2},
$$

befriedigen, und somit der Gleichung

$$
(x-a)^{6}-4 a(x-a)^{5}+10 b(x-a)^{3}-c(x-a)+5 b^{2}-a c=0
$$

genügen, deren Coefficienten auf rationalem Wege von nur zwei Parametern abhängig gemacht werden können.

Diese Gleichung definiert also eine Hilfsfunction von zwei Variabeln, mit deren Hilfe die Wurzeln der allgemeinen Gleichung fünften Grades sich rational ausdrücken lassen. K r o necker sicht sie als Normalresolvente an.

Wir wollen nach Brioschi's Vorgang zeigen, wie man rationale Funetionen der Gleichung fünften Grades bilden kann, welche die Relationen (2) befriedigen.

Zuerst bemerken wir, dass die alternierende Gruppe sich aus den Substitutionen

$$
|v, v+1| \quad|v, 4 v| \quad\left|v, 3 v^{3}\right|
$$

zusammensetzt. Es möge nun $v$ eine cyklische Function der Wurzeln der Gleichung fünften Grades, und $v^{1}$ deren Wert, nach Anwendung der Substitution $|\nu, 4 \nu|$ sein. Bezeichnet man die Differenz $v-v^{1}$ mit $u_{\infty}$, und deren Wert nach Anwendung von $\left|v, 3 v^{3}+n\right|$ mit $u_{n}$, so sieht man, dass die Substitution $|v, v+1|, u_{n}$ in $u_{n+1}$, die Substitution $|v, 4 \nu|, u_{n}$ in $-u_{4 n}$, die Substitution $\left|v, 3 v^{2}\right|, u_{\infty}, u_{0}$, $u_{1}, u_{2}, u_{3}, u_{4}$ in $u_{0}, u_{\infty},-1 u_{1}, u_{3}, u_{2},-u_{4}$ verwandelt.

Betrachten wir die Function:

$$
U_{\infty}=t_{\infty} u_{\infty}+t_{0} u_{0}+t_{1} u_{1}+t_{2} u_{2}+t_{3} u_{3}+t_{4} u_{4},
$$


so erhalten wir nach Anwendung von $\left|\nu, 3 \nu^{3}+n\right|$

$$
\begin{aligned}
& U_{0}=t_{\infty} u_{0}+t_{0} u_{\infty}-t_{1} u_{1}+t_{2} u_{3}+t_{3} u_{2}-t_{4} u_{4} \\
& U_{1}=t_{\infty} u_{1}+t_{0} u_{\infty}-t_{1} u_{2}+t_{2} u_{4}+t_{3} u_{3}-t_{4} u_{0} \\
& U_{2}=t_{\infty} u_{2}+t_{0} u_{\infty}-t_{1} u_{3}+t_{2} u_{0}+t_{3} u_{4}-t_{4} u_{1} \\
& U_{3}=t_{\infty} u_{3}+t_{0} u_{\infty}-t_{1} u_{4}+t_{2} u_{1}+t_{3} u_{0}-t_{4} u_{3} \\
& U_{4}=t_{\infty} u_{4}+t_{0} u_{\infty}-t_{1} u_{0}+t_{2} u_{2}+t_{3} u_{1}-t_{4} u_{3} .
\end{aligned}
$$

Setzt man nun

$$
\begin{gathered}
x_{\infty}=U_{\infty}^{2}, x_{r}=U_{r}^{2}, t_{\infty}=\sqrt{5}, t_{r}=1 \quad(r=0,1,2,3,4) \\
a_{0} \sqrt{5}=u_{\infty} \sqrt{5}+u_{0}+u_{1}+u_{2}+u_{3}+u_{4} \\
\frac{1}{2} a_{1} \sqrt{5}=u_{0}+\varepsilon^{4} u_{1}+\varepsilon^{3} u_{2}+\varepsilon^{2} u_{3}+\varepsilon u_{4} \\
\frac{1}{2} a_{2} \sqrt{5}=u_{0}+\varepsilon u_{1}+\varepsilon^{2} u_{2}+\varepsilon^{3} u_{3}+\varepsilon^{4} u_{4},
\end{gathered}
$$

so genügen die Größen $x$ den Gleichungen (1) und (2); und da eine symmetrische Function der $x$ nach Anwendung der Substitutionen unverändert bleibt, so sind die Größjen $a, b, c$ nunmehr rational in den Coefficienten der Gleichung fünften Grades und der Quadratwurzel aus ihrer Discriminante.

Es ergibt sich mittelst (1) und (4), dass nicht nur die Function

$$
y_{r}=\left(x_{\infty}-x_{r}\right)\left(x_{r+2}-x_{r+3}\right)\left(x_{r+4}-x_{r+1}\right) \text {, }
$$

sondern auch ihre Qudratwurzel eine rationale Function der Wurzeln der Gleichung fünften Grades ist, und somit zu einer rationalen Resolvente Anlass gibt, wie wir bei der Besprechung der Brioschischen Arbeit erwähnten. Wegen der engen Beziehung zu den Transformationsgleichungen gestattet die Gleichung (2) eine leichte Anwendung der elliptischen Functionen auf die Gleichung fünften Grades. Für die Multiplicatorgleichung war, wie wir sahen,

$$
a=1 \quad b=0 \quad c=-2^{8} k^{8} k^{\prime 2},
$$

Kronecker machte auf den Fall $a=0$ aufmerksam, der durch die Einführung einer accessorischen Quadratwurzel erhalten werden kann.

Setzt man $v=v_{1}+v v_{2}$ wo $v_{1}, v_{2}$ irgendwelche zwei cyklische Functionen sind, so zeigt die Gleichung

$$
a=a_{0}^{2}+a_{1} a_{2}=0,
$$

dass $v$ durch eine quadratische Gleichung bestimmt ist, deren Coefficienten rational in den Coefficienten der Gleichung fünften Grades und der Quadratwurzel aus ihrer Discriminante sind. 
Eine entsprechende Transformationsgleichung ist: ${ }^{1}$ )

$$
z^{5}-\frac{160}{k^{2} k^{\prime 2}} z^{3}-\frac{2^{8}\left(1-16 k^{8} k^{\prime 2}\right)}{k^{4} k^{\prime 4}} z+\frac{2^{5} \cdot 5}{k^{4} k^{14}}=0,
$$

wo

$$
z=\frac{1}{M}\left(\sqrt{\frac{\lambda}{k}}+\sqrt{\frac{\lambda^{\prime}}{k^{\prime}}}\right)^{2}=\left(\frac{c n 2 \omega}{c n 4 \omega}-\frac{c n 4 \omega}{c n 2 \omega}\right)
$$

ist.

Die Vergleichung mit (2) liefert schließlich

$$
a=0, \quad b=-\frac{16}{k^{2} k^{\prime 2}}, \quad c=2^{8} \frac{1-16 k^{2} k^{\prime 2}}{k^{4} k^{\prime 4}} .
$$

1) Siehe Seite 53. 